TRANSACTIONS OF THE

AMERICAN MATHEMATICAL SOCIETY

Volume 362, Number 1, January 2010, Pages 357-388

S 0002-9947(09)04873-9

Article electronically published on July 24, 2009

\title{
SECOND ANGULAR DERIVATIVES AND PARABOLIC ITERATION IN THE UNIT DISK
}

\author{
MANUEL D. CONTRERAS, SANTIAGO DÍAZ-MADRIGAL, \\ AND CHRISTIAN POMMERENKE
}

\begin{abstract}
In this paper we deal with second angular derivatives at DenjoyWolff points for parabolic functions in the unit disc. Namely, we study and analyze the existence and the dynamical meaning of this second angular derivative. For instance, we provide several characterizations of that existence in terms of the so-called Koenigs function. It is worth pointing out that there are two quite different classes of parabolic iteration: those with positive hyperbolic step and those with zero hyperbolic step. In the first case, the Koenigs function is in the Carathéodory class but, in the second case, it is even unknown if it is normal. Therefore, the ideas and techniques to approach these two cases are really different. In the end, we also present several rigidity results related to the second angular derivatives at Denjoy-Wolff points.
\end{abstract}

\section{INTRODUCTION}

The dynamical properties of a holomorphic self-map of the unit disk $\varphi \in$ $\operatorname{Hol}(\mathbb{D} ; \mathbb{D})$ have attracted considerable interest since the beginning of the last century. Now it is known that a major role is played by the collection of all inner and boundary fixed points of $\varphi$. We recall that a point $b \in \mathbb{D}$ is said to be an inner fixed point of $\varphi$ if $\varphi(b)=b$; likewise, a point $b \in \partial \mathbb{D}$ is said to be a boundary fixed point of $\varphi$ if $\lim _{r \rightarrow 1} \varphi(r b)=b$.

In a natural way, the usual classification of the members of $\operatorname{Hol}(\mathbb{D} ; \mathbb{D})$ takes into account the above set of fixed points. For instance, $\varphi \in \operatorname{Hol}(\mathbb{D} ; \mathbb{D})$ is said to be elliptic if $\varphi$ has at least one inner fixed point. It is a deep result, the socalled Denjoy-Wolff Theorem, that any non-elliptic map $\varphi \in \operatorname{Hol}(\mathbb{D} ; \mathbb{D})$ always has a boundary fixed point $\tau$ with a very strong property: all of their forward orbits tend to $\tau$. This point is clearly unique and it is called the Denjoy-Wolff point of $\varphi$. Moreover, $\varphi$ is somehow differentiable at $\tau$. Since $\tau$ belongs to the boundary of the unit disk, this comment requires a clarification and this leads us to recall the concept of angular limit.

Received by the editors December 9, 2005 and, in revised form, February 20, 2008.

2000 Mathematics Subject Classification. Primary 30D05, 32H40; Secondary 32H50.

Key words and phrases. Second angular derivative, parabolic functions, Denjoy-Wolff point, Koenigs function, rigidity.

This research has been partially supported by the Ministerio de Ciencia y Tecnologia and the European Union (FEDER) project MTM2006-14449-C02-01 and by La Consejería de Educación y Ciencia de la Junta de Andalucía. 
Let $f: \mathbb{D} \rightarrow \mathbb{C}$ be an analytic function and $b \in \partial \mathbb{D}$. It is said that $L \in \mathbb{C}_{\infty}$ is the angular limit of $f$ in $b$ when $z$ tends to $b$ if, for every $\alpha \in(0, \pi / 2)$,

$$
\lim _{z \in S(b, \alpha),} f(z)=L,
$$

where $S(b, \alpha)$ denotes the Stolz angle centered in $b$ and with opening $\alpha$. The number $L$ is more commonly denoted by $\angle \lim _{z \rightarrow b} f(z)$. If $L$ is finite and the angular limit

$$
M:=\angle \lim _{z \rightarrow b} \frac{f(z)-L}{z-b} \in \mathbb{C}_{\infty}
$$

exists, then $f$ is said to be angular differentiable at $b$ and we write $f^{\prime}(b)=M$.

Turning to iteration, if $\tau$ is the Denjoy-Wolff point of some non-elliptic $\varphi \in$ $\operatorname{Hol}(\mathbb{D} ; \mathbb{D})$, then $\varphi$ is angular differentiable at $\tau$ and $\varphi^{\prime}(\tau) \in(0,1]$. We recall that $\varphi$ is said to be parabolic whenever $\varphi^{\prime}(\tau)=1$ and, otherwise, hyperbolic.

Hyperbolic iteration in the disk is quite well understood now (see for instance the papers [10] and 3] and the references therein). However, in the parabolic case, many interesting dynamical questions remain to be answered. Bearing in mind the importance of first order angular differentiability in what is known in both cases, we have proposed here to study the dynamical behavior of parabolic iteration assuming some kind of high angular differentiability at the corresponding Denjoy-Wolff point. In a certain sense and for different purposes (composition operators [2], rigidity results [13, [14, ...), this approach has already been considered by several authors and, indeed, some of our results are non-trivial extensions of some of their results. Concerning the remarkable paper [2, we want to underline that their results and techniques have been an important source of inspiration for this work. Anyway, most of our results are, as far as we know, really new and we think this is the first paper dealing explicitly with general questions about the existence or dynamical meaning of the second angular derivative.

Since the concepts of angular differentiability of high order are intuitive but certainly technical, we have decided to group them all together in the next section. As usual, we make good use in our proofs of the pseudo-hyperbolic distance $\widetilde{\rho}_{\mathbb{D}}\left(z_{1}, z_{2}\right)$ in the unit disk as well as the hyperbolic distance $\rho_{\mathbb{D}}\left(z_{1}, z_{2}\right)$. Sometimes, this will be done indirectly. We mean by this that we will prefer to work with $\widetilde{\rho}_{\mathbb{H}}$ and $\rho_{\mathbb{H}}$, respectively, the pseudo-hyperbolic distance and the hyperbolic distance in the right half-plane $\mathbb{H}$. In this respect, we recall that a holomorphic function $f: \mathbb{H} \rightarrow \mathbb{C}$ has angular limit $L \in \mathbb{C}_{\infty}$ at the point $\infty$, whenever, for every $c>0$,

$$
\lim _{w \in S(\infty, c), w \rightarrow \infty} f(w)=L,
$$

where $S(\infty, c)=\{u+i v \in \mathbb{H}:|v| \leq c u\}$. As before, the number $L$ will be denoted by $\angle \lim _{w \rightarrow \infty} f(w)$.

The paper is divided into six sections, apart from this introduction. In the next section, we present several not so widely known definitions and several minor results (most of them only partially known) concerning angular differentiability of arbitrary order at a boundary fixed point. In section three, we recall, also giving new comments, the main types of classification of parabolic maps and give a short review of the intertwining maps used in parabolic iteration. In addition, we prove several technical lemmas that we will need repeatedly in the remaining sections. In a certain sense, sections two and three can be thought of as preparatory sections. The other four sections are really the core of the paper. Namely, in section four, we 
treat the existence and dynamical meaning of second order angular differentiability at Denjoy-Wolff points for parabolic maps with positive hyperbolic step. Sections five and six are devoted to parabolic maps with zero hyperbolic step. Basically, we present some general results for those functions in section five and treat the corresponding problems related to second order angular differentiability in the sixth section. Finally, in the last section, we present several rigidity results when second order angular derivatives vanish at Denjoy-Wolff points of non-elliptic maps.

\section{High order ANGUlar DifFERENTIABILITy at BOUNDARY FIXED POINTS}

Consider a map $\varphi \in \operatorname{Hol}(\mathbb{D} ; \mathbb{C})$ with a boundary fixed point $b \in \partial \mathbb{D}$. We say that $\varphi$ is of angular-class of order $p \in \mathbb{N}$ at $b$, and we denote it by $\varphi \in C_{A}^{p}(b)$, if

$$
\varphi(z)=b+\sum_{j=1}^{p} \frac{a_{j}}{j !}(z-b)^{j}+\gamma(z), \quad z \in \mathbb{D},
$$

where $a_{1}, \ldots, a_{p} \in \mathbb{C}$ and $\gamma \in \operatorname{Hol}(\mathbb{D} ; \mathbb{C})$ with

$$
\angle \lim _{z \rightarrow b} \frac{\gamma(z)}{(z-b)^{p}}=0
$$

It is clear that the numbers $a_{1}, \ldots, a_{p}$ appearing in the above expression are necessarily unique.

According to the Julia-Carathéodory Theorem, we easily see that $\varphi \in C_{A}^{1}(b)$ if and only if $\varphi$ has a finite angular derivative at $b$. Clearly, when this happens, the corresponding number $a_{1}$ is exactly $\varphi^{\prime}(b)$. In particular, parabolic and hyperbolic members of $\operatorname{Hol}(\mathbb{D} ; \mathbb{D})$ are of angular-class of first order at their Denjoy-Wolff points.

It is also straightforward to check that $\varphi$ belongs to $C_{A}^{2}(b)$ if and only if $\varphi \in C_{A}^{1}(b)$ and the following angular limit exists finitely:

$$
L:=\angle \lim _{z \rightarrow b} \frac{\varphi(z)-b-\varphi^{\prime}(b)(z-b)}{(z-b)^{2}} .
$$

Now the corresponding numbers $a_{1}$ and $a_{2}$ are just $a_{1}=\varphi^{\prime}(b)$ and $a_{2}=2 L$. In what follows, $a_{2}$ will be written as $\varphi^{\prime \prime}(b)$. Note that if $\varphi$ is parabolic with Denjoy-Wolff point $\tau \in \partial \mathbb{D}$, then $\varphi \in C_{A}^{2}(\tau)$ if and only if the following angular limit exists finitely:

$$
\angle \lim _{z \rightarrow \tau} \frac{\varphi(z)-z}{(z-\tau)^{2}} .
$$

It is important to underline that there are examples showing that being of angular-class of order two at a certain boundary fixed point is much stronger than being of angular-class of order one. For instance, let us consider

$$
\varphi(z):=\frac{2 z+(1-z) \log \left(\frac{2}{1-z}\right)}{2+(1-z) \log \left(\frac{2}{1-z}\right)}, z \in \mathbb{D},
$$

where $\log$ is the principal branch of the logarithm. It is possible to check that $\varphi$ is a parabolic holomorphic self-map of the unit disk having the point 1 as Denjoy-Wolff point. Trivially, $\varphi \in C_{A}^{1}(1)$. However, $\varphi \notin C_{A}^{2}(1)$ since

$$
\angle \lim _{z \rightarrow 1} \frac{\varphi(z)-z}{(z-1)^{2}}=\lim _{z \rightarrow 1} \frac{\varphi(z)-z}{(z-1)^{2}}=\infty .
$$


In the proofs of our results, we often pass to the right half-plane $\mathbb{H}$. As usual, given a non-elliptic map $\varphi \in \operatorname{Hol}(\mathbb{D} ; \mathbb{D})$ with Denjoy-Wolff point $\tau \in \partial \mathbb{D}$, we call $\phi:=\sigma_{\tau} \circ \varphi \circ\left(\sigma_{\tau}\right)^{-1}$ the iteration map associated with $\varphi$ in $\mathbb{H}$, where $\sigma_{\tau}$ is the usual Cayley map related to $\tau$, that is,

$$
\sigma_{\tau}(z):=\frac{\tau+z}{\tau-z}, \quad z \in \mathbb{D}
$$

It is well known (see 11 for more details) that $\phi \in \operatorname{Hol}(\mathbb{H} ; \mathbb{H})$ and it has $\infty$ as Denjoy-Wolff point. Moreover, the corresponding angular derivative at $\infty$ satisfies that $\phi^{\prime}(\infty)=\varphi^{\prime}(\tau)^{-1} \in[1,+\infty)$ and, indeed,

$$
\angle \lim _{w \rightarrow \infty} \frac{\phi(w)-\varphi^{\prime}(\tau)^{-1} w}{w}=0 .
$$

Quite useful for the aim of this paper is the following result which links second order differentiability of $\varphi$ at its Denjoy-Wolff point and certain properties of the associated iteration map in $\mathbb{H}$. The proof basically involves quite standard computations with Cayley maps. In fact, with the same notation as in the proposition, the key is the following identity:

$$
\frac{\varphi(z)-\tau-\alpha(z-\tau)}{(z-\tau)^{2}}=\frac{\bar{\tau} \alpha}{2} \frac{w+1}{\phi(w)+1}\left[\phi(w)-\frac{1}{\alpha} w+1-\frac{1}{\alpha}\right],
$$

where $w=\sigma_{\tau}(z)$.

Proposition 2.1. Let $\varphi \in \operatorname{Hol}(\mathbb{D} ; \mathbb{D})$ be non-elliptic with Denjoy-Wolff point $\tau \in$ $\partial \mathbb{D}$ and let $\phi$ be the associated iteration map in $\mathbb{H}$. Write $\alpha:=\varphi^{\prime}(\tau) \in(0,1]$. Then, the map $\varphi$ belongs to $C_{A}^{2}(\tau)$ if and only if the following angular limit exists finitely:

$$
a:=\angle \lim _{w \rightarrow \infty}\left(\phi(w)-\frac{1}{\alpha} w\right)
$$

that is, for every $w \in \mathbb{H}$,

$$
\phi(w)=\frac{1}{\alpha} w+a+\gamma(w),
$$

where $\gamma \in \operatorname{Hol}(\mathbb{H} ; \mathbb{C})$ with $\angle \lim _{w \rightarrow \infty} \gamma(w)=0$.

Moreover, when the above statements hold, we have that $\operatorname{Re}(a) \geq 0$ and

$$
\varphi^{\prime \prime}(\tau) \tau=\alpha^{2} a+\alpha(\alpha-1) .
$$

In the final part of the paper, we will also consider third order angular differentiability at Denjoy-Wolff points and this will require a variant of the above proposition for that third order. Once again, the proof of the corresponding result involves computations with Cayley maps and, now, the corresponding key identity is

$$
\frac{\varphi(z)-\tau-\alpha(z-\tau)-\frac{\varphi^{\prime \prime}(\tau)}{2}(z-\tau)^{2}}{(z-\tau)^{3}}=\frac{\bar{\tau}}{4} \frac{w+1}{\phi(w)+1}(A-B+C),
$$

where $w=\sigma_{\tau}(z)$ and

$$
\begin{aligned}
A & =\left(\varphi^{\prime \prime}(\tau)-\bar{\tau} \alpha\right)\left(\phi(w)-\frac{1}{\alpha} w\right), \\
B & =\bar{\tau} \alpha\left(w \phi(w)-\frac{1}{\alpha} w^{2}-a w\right), \\
C & =\varphi^{\prime \prime}(\tau)-\bar{\tau}(\alpha-1) .
\end{aligned}
$$


Proposition 2.2. Let $\varphi \in \operatorname{Hol}(\mathbb{D} ; \mathbb{D})$ be non-elliptic with Denjoy-Wolff point $\tau \in$ $\partial \mathbb{D}$ and let $\phi$ be the associated iteration map in $\mathbb{H}$. Denote $\alpha:=\varphi^{\prime}(\tau) \in(0,1]$ and assume that $\varphi$ belongs to $C_{A}^{2}(\tau)$. Then, the map $\varphi$ belongs to $C_{A}^{3}(\tau)$ if and only if there exist two complex numbers $a, b$ such that, for every $w \in \mathbb{H}$,

$$
\phi(w)=\frac{1}{\alpha} w+a+\frac{b}{w}+\gamma(w),
$$

where $\gamma \in \operatorname{Hol}(\mathbb{H} ; \mathbb{C})$ with $\angle \lim _{w \rightarrow \infty} w \gamma(w)=0$.

Moreover, when the above statements hold, we have that

$$
\varphi^{\prime \prime \prime}(\tau) \tau^{2}=\frac{3}{2} \alpha\left[(a \alpha+\alpha-1)^{2}-b \alpha\right] .
$$

If, in addition, $\operatorname{Re} a=0$, then $\operatorname{Re} b \geq 0$.

As is indicated in [13] and in 2] for the parabolic case, the expression of the above number $b$ is quite related to what can be considered the angular Schwarzian derivative $S_{\varphi}(\tau)$ of $\varphi$ at the corresponding Denjoy-Wolff point. Indeed, this fact is true in the general non-elliptic case. Namely, using the above proposition and bearing in mind the algebraic rules for computing angular derivatives, it can be checked that

$$
b=-\frac{2}{3} \frac{\tau^{2}}{\alpha}\left[\left(\frac{\varphi^{\prime \prime}(\tau)}{\varphi^{\prime}(\tau)}\right)^{\prime}-\frac{1}{2}\left(\frac{\varphi^{\prime \prime}(\tau)}{\varphi^{\prime}(\tau)}\right)^{2}\right]=-\frac{2}{3} \frac{\tau^{2}}{\alpha} S_{\varphi}(\tau) .
$$

\section{Classification of PARABOlic iteration AND INTERTWining MAPS}

Given a parabolic map $\varphi \in \operatorname{Hol}(\mathbb{D} ; \mathbb{D})$, we say that $\varphi$ is of zero hyperbolic step (in short, zero h-step) if, for some $z_{0} \in \mathbb{D}, \rho_{\mathbb{D}}\left(z_{n}, z_{n+1}\right) \stackrel{n}{\rightarrow} 0$, where $z_{n}=\varphi_{n}\left(z_{0}\right)$. It is well known that the word "some" can be replaced here by "all". In other words, the definition does not depend on the chosen initial point of the orbit. Using the Scharwz-Pick Lemma, the parabolic maps which are not of zero h-step are those $\varphi \in \operatorname{Hol}(\mathbb{D} ; \mathbb{D})$ such that

$$
\lim _{n} \rho_{\mathbb{D}}\left(z_{n}, z_{n+1}\right)>0,
$$

for some (resp. all) forward orbit $\left(z_{n}\right)$ of $\varphi$. That is the reason they are said to be of positive hyperbolic step (in short, positive h-step).

It is worth mentioning that this way of classifying parabolic maps loses its meaning when we consider the above limits only in the angular sense. This is the content of the next lemma, which will be used in section five. The explanation of this apparently paradoxical fact is that, in the positive h-step case, all the forward orbits tend tangentially to the Denjoy-Wolff point [9, Remark 1].

Lemma 3.1. Let $\varphi \in \operatorname{Hol}(\mathbb{D} ; \mathbb{D})$ be parabolic and $\tau \in \partial \mathbb{D}$ the corresponding DenjoyWolff point. Then $\angle \lim _{z \rightarrow \tau} \rho_{\mathbb{D}}(\varphi(z), z)=0$.

Proof. As usual, we prove the above statement for the right half-plane $\mathbb{H}$ and we denote by $\phi$ the iteration map in $\mathbb{H}$ associated with $\varphi$. We recall that $\phi \in \mathrm{Hol}(\mathbb{H} ; \mathbb{H})$ and it is a parabolic map with $\infty$ as Denjoy-Wolff point. Write $w_{n}=x_{n}+i y_{n}=$ $\phi_{n}(1)$. We note that

$$
\widetilde{\rho}_{\mathbb{H}}(w, \phi(w))=\left|\frac{\phi(w)-w}{\phi(w)+\bar{w}}\right|=\frac{\left|\frac{\phi(w)-w}{\operatorname{Re} w}\right|}{\left|\frac{\phi(w)-w}{\operatorname{Re} w}+2\right|} .
$$


Moreover, by [9, Theorem 2], we have that

$$
\angle \lim _{w \rightarrow \infty} \frac{\phi(w)}{w}=1 .
$$

Now, fix $0<c<+\infty$ and consider the Stolz angle with respect to $\infty, S(\infty, c)=$ $\{u+i v:|v|<c u\}$. If $w=u+i v \in S(\infty, c)$, then

$$
\left|\frac{\phi(w)-w}{\operatorname{Re} w}\right|=\left|\frac{\phi(w)-w}{w}\right|\left|\frac{w}{\operatorname{Re} w}\right| \leq(1+c)\left|\frac{\phi(w)}{w}-1\right| .
$$

Therefore, $\lim _{w \rightarrow \infty, w \in S(\infty, c)} \frac{\phi(w)-w}{\operatorname{Re} w}=0$. Looking at the above expression for $\widetilde{\rho}_{\mathbb{H}}(w, \phi(w))$, we conclude that $\angle \lim _{w \rightarrow \infty} \widetilde{\rho}_{\mathbb{H}}(w, \phi(w))=0$ as wanted.

It is also well known that if $z_{0} \in \mathbb{D}$ and $\varphi \in \operatorname{Hol}(\mathbb{D} ; \mathbb{D})$ is parabolic with Denjoy-Wolff point $\tau \in \partial \mathbb{D}$, then the sequence of real numbers $\left(\operatorname{Re} \phi_{n}\left(w_{0}\right)\right)$ is nondecreasing, where $\phi$ is the iteration map associated with $\varphi$ in $\mathbb{H}$ and $w_{0}:=\sigma_{\tau}\left(z_{0}\right)$. Therefore, we can consider $L\left(z_{0}\right):=\lim _{n} \operatorname{Re} \phi_{n}\left(w_{0}\right) \in(0,+\infty]$. Whenever $L\left(z_{0}\right) \neq$ $+\infty$, the map $\varphi$ is said to be of finite shift and, otherwise, of infinite shift with respect to the point $z_{0}$. It is just a computation to check that the forward orbit associated with $z_{0}$ of $\varphi$ is of finite shift type if and only if

$$
\sup _{n} \frac{1-\left|\varphi_{n}\left(z_{0}\right)\right|^{2}}{\left|\tau-\varphi_{n}\left(z_{0}\right)\right|^{2}}<+\infty
$$

Our first result shows that, indeed, this definition does not depend on the initial point of the orbit, either. We provide a proof since we are not aware of any reference for it.

Proposition 3.2. Let $\varphi \in \operatorname{Hol}(\mathbb{D} ; \mathbb{D})$ be parabolic with Denjoy-Wolff point $\tau \in \partial \mathbb{D}$. If a forward orbit of $\varphi$ is of finite shift type, then all of the forward orbits of $\varphi$ are also of finite shift type. In fact, for any two arbitrary points $z_{1}, z_{2} \in \mathbb{D}$, the following limit always exists and is positive:

$$
L:=\left(L\left(z_{1}, z_{2}\right)=\right) \lim _{n} \frac{1-\left|\varphi_{n}\left(z_{1}\right)\right|^{2}}{\left|\tau-\varphi_{n}\left(z_{1}\right)\right|^{2}} \frac{\left|\tau-\varphi_{n}\left(z_{2}\right)\right|^{2}}{1-\left|\varphi_{n}\left(z_{2}\right)\right|^{2}}>0 .
$$

Moreover, if $\varphi$ is of zero hyperbolic step, then $L=1$.

Proof. As usual, we consider and prove the above statement for the right half-plane $\mathbb{H}$. In this sense, we denote by $\phi$ the iteration map in $\mathbb{H}$ associated with $\varphi$. We point out that

$$
\operatorname{Re} \phi_{n}(w)=\frac{1-\left|\varphi_{n}(z)\right|^{2}}{\left|\tau-\varphi_{n}(z)\right|^{2}},
$$

where $\sigma_{\tau}(z)=w$. According to $[9$, we know that the limit

$$
h(w):=\lim _{n} \frac{\phi_{n}(w)-i y_{n}}{x_{n}}, \quad x_{n}=\operatorname{Re} \phi_{n}(1), y_{n}=\operatorname{Im} \phi_{n}(1),
$$

exists, for every $w \in \mathbb{H}$. Moreover, if $\phi$ is of zero h-step, then $h$ is constant and equal to one and if $\phi$ is of positive h-step, then $h \in \operatorname{Hol}(\mathbb{H} ; \mathbb{H})$. Now, take an arbitrary point $w_{1} \in \mathbb{H}$. Therefore, if $\phi$ is of zero h-step,

$$
\lim _{n} \frac{\operatorname{Re} \phi_{n}\left(w_{1}\right)}{x_{n}}=1
$$


and, if $\phi$ is of positive h-step,

$$
\lim _{n} \frac{\operatorname{Re} \phi_{n}\left(w_{1}\right)}{x_{n}}=\operatorname{Reh}\left(w_{1}\right)>0 .
$$

From this, the result is clear.

Combining the concepts of zero/positive h-step and finite/infinite shift type, we have a priori a classification of parabolic maps in four different classes. However, we really only have three. This fact has been observed by P. Poggi-Corradini [8]. Anyway, for further reference and for the sake of completeness, we present here a slight variation of his proof.

Proposition 3.3. Let $\varphi \in \operatorname{Hol}(\mathbb{H} ; \mathbb{H})$ be parabolic of zero hyperbolic step. Then $\varphi$ is of infinite shift type.

Proof. Again, we consider and prove the above statement for the right half-plane $\mathbb{H}$. Let $\phi$ be the analytic map in $\mathbb{H}$ associated with $\varphi$. We know now (Proposition 3.2) that being of finite/infinite shift type does not depend on the initial point. So, suppose on the contrary, that

$$
\lim _{n} x_{n}=L \in(0,+\infty),
$$

where $x_{n}=\operatorname{Re} \phi_{n}(1)$ and $y_{n}=\operatorname{Im} \phi_{n}(1)$. According again to [9], we have that

$$
h_{n}(w)=\frac{\phi_{n}(w)-i y_{n}}{x_{n}} \stackrel{n}{\longrightarrow} 1, \quad \text { for every } w \in \mathbb{H} .
$$

Therefore, $\left(\operatorname{Re} \phi_{n}(w)\right)$ tends also to $L$, independently of the initial point $w \in \mathbb{H}$. Since $\left(\operatorname{Re} \phi_{n}(w)\right)$ is non-decreasing, also for every $w \in \mathbb{H}$, we arrive at a contradiction just by noting that

$$
L+1 \leq \operatorname{Re} \phi_{n}(L+1) \stackrel{n}{\rightarrow} L .
$$

The other three classes are non-empty. In fact, $\phi(w)=w+1$ is an example of a parabolic map in $\mathbb{H}$ with zero h-step and infinite shift type and $\phi(w)=w+i$ is an example of a parabolic map in $\mathbb{H}$ with positive h-step and finite shift type. Besides, examples of parabolic maps in $\mathbb{H}$ with positive h-step and infinite shift type are given by

$$
\phi(w)=(\sqrt{w}+a(1+i))^{2} \text {, with } a>0 \text { and } w \in \mathbb{H} .
$$

According to Theorem 4.1 below, any parabolic map of this type (positive hstep and infinite shift type) provides an example of separation (with respect to the Denjoy-Wolff point) between being of angular-class of order two and being of angular-class of order one.

Tangential/non-tangential convergence, zero/positive h-step and finite/infinite shift type are concepts which are known to be deeply connected for the important and basic family of parabolic linear fractional self-maps in the unit disk. In the next result and, for the sake of clarity, we have put together some of these characterizations. In a certain sense, a large part of our work in sections four and five is modelled upon this result.

Proposition 3.4. Let $\varphi \in \operatorname{Hol}(\mathbb{D} ; \mathbb{D})$ be a parabolic linear fractional map with Denjoy-Wolff point $\tau \in \partial \mathbb{D}$. Then $\varphi$ is analytic beyond the closed unit disk and $\varphi^{\prime \prime}(z) \neq 0$, for every $z \in \mathbb{D}$. Moreover, the following statements are equivalent. 
(1) $\varphi \in \operatorname{Aut}(\mathbb{D})$.

(2) The map $\varphi$ is of positive hyperbolic step.

(3) The map $\varphi$ is of finite shift.

(4) $\operatorname{Re}\left(\varphi^{\prime \prime}(\tau) \tau\right)=0$.

(5) Every (resp. some) forward orbit of $\varphi$ tends tangentially to $\tau$.

It is worth mentioning that, by Proposition 2.1, the opposite statement of the above item 4 is $\operatorname{Re}\left(\varphi^{\prime \prime}(\tau) \tau\right)>0$. We also want to point out that item 5 just says that any forward orbit $\left(z_{n}\right)$ of $\varphi$ cannot be asymptotically contained in any Stolz angle of $\tau$. However, it is also known that, for all the forward orbits $\left(z_{n}\right)$ of these parabolic linear fractional self-maps of positive h-step, we have that the limits

$$
\lim _{n} \operatorname{Arg}\left(1-\bar{\tau} z_{n}\right)
$$

always exist, all of them have the same value and this value is $\frac{\pi}{2}$ or $-\frac{\pi}{2}$.

In our analysis of second angular differentiability, an important role is played by the intertwining maps developed by the third author alone 9 and jointly with Baker [1. Namely, given a parabolic map $\phi \in \operatorname{Hol}(\mathbb{H} ; \mathbb{H})$ with Denjoy-Wolff point $\infty$ and of positive h-step, it is proved in [9] that the limit

$$
h(w):=\lim _{n} \frac{\phi_{n}(w)-i y_{n}}{x_{n}}, \quad w \in \mathbb{H}, \quad x_{n}=\operatorname{Re} \phi_{n}(1), y_{n}=\operatorname{Im} \phi_{n}(1)
$$

exists uniformly on compact subsets of $\mathbb{H}$. Moreover, $h \in \operatorname{Hol}(\mathbb{H} ; \mathbb{H})$ and it satisfies

$$
h \circ \phi=h+i \delta
$$

for a certain non-zero real number $\delta=\delta(\phi, 1)=\lim _{n} \frac{y_{n+1}-y_{n}}{x_{n}}$. In other words, $h$ is an intertwining map between the iteration couples $(\phi, \mathbb{H})$ and $(w+i \delta, \mathbb{H})$. In what follows, this function $h$ will be called the Koenigs map of $\phi$ (normalized with respect to 1). Clearly, we can normalize with respect to another point $w_{0} \in \mathbb{H}$. In this case,

$$
\begin{aligned}
h_{w_{0}}(w) & =\lim _{n} \frac{\phi_{n}(w)-i \operatorname{Im} \phi_{n}\left(w_{0}\right)}{\operatorname{Re} \phi_{n}\left(w_{0}\right)} \\
& =\lim _{n}\left(\frac{\phi_{n}(w)-i y_{n}}{x_{n}}-i \frac{\operatorname{Im} \phi_{n}\left(w_{0}\right)-y_{n}}{x_{n}}\right) \frac{x_{n}}{\operatorname{Re} \phi_{n}\left(w_{0}\right)} \\
& =\frac{h(w)-i \operatorname{Im} h\left(w_{0}\right)}{\operatorname{Re} h\left(w_{0}\right)}=\frac{h(w)-i \operatorname{Im} h\left(w_{0}\right)}{\operatorname{Re} h\left(w_{0}\right)} .
\end{aligned}
$$

Therefore, the corresponding conjugation equation is now $h_{w_{0}} \circ \phi=h_{w_{0}}+i \frac{\delta}{\operatorname{Reh}\left(w_{0}\right)}$. We note that the signs of $\frac{\delta}{\operatorname{Reh}\left(w_{0}\right)}$ and $\delta$ are exactly the same. It is important to isolate this fact and we say that the above parabolic of positive h-step map $\phi$ is of type I whenever $\delta>0$ and of type II if $\delta<0$. All of these definitions pass to the unit disk context in the usual way by using Cayley maps. For instance, given an arbitrary parabolic map $\varphi \in \operatorname{Hol}(\mathbb{D} ; \mathbb{D})$ with Denjoy-Wolff point $\tau \in \partial \mathbb{D}$ and of positive h-step, the corresponding Koenigs map of $\varphi$ will be $h_{\mathbb{D}}:=h \circ \sigma_{\tau}$, where $h$ is the Koenigs map of $\phi$, that is, the analytic map in $\mathbb{H}$ associated with $\varphi$. 
Given a parabolic map $\phi \in \operatorname{Hol}(\mathbb{H} ; \mathbb{H})$ with Denjoy-Wolff point $\infty$ and of zero hyperbolic step, it is proved in [1] that the limit

$$
h(w):=\lim _{n} \frac{\phi_{n}(w)-w_{n}}{w_{n+1}-w_{n}}, \quad w \in \mathbb{H}, \quad w_{n}=\phi_{n}(1)
$$

exists uniformly on compact subsets of $\mathbb{H}$. Thus, $h \in \operatorname{Hol}(\mathbb{H} ; \mathbb{C})$ and, in general, that is all we can say about the image of $h$. In what follows, this function $h$ will also be called the Koenigs map of $\phi$ (normalized with respect to 1 ) and it satisfies

$$
h \circ \phi=h+1 .
$$

In other words, $h$ is an intertwining map between the iteration couples $(\phi, \mathbb{H})$ and $(w+1, \mathbb{C})$. Again, these definitions pass to the unit disk context in the usual way. So, given an arbitrary parabolic map $\varphi \in \operatorname{Hol}(\mathbb{D} ; \mathbb{D})$ with Denjoy-Wolff point $\tau \in \partial \mathbb{D}$ and of zero h-step, the corresponding Koenigs function of $\varphi$ will be $h_{\mathbb{D}}=h \circ \sigma_{\tau}$, where $h$ is the Koenigs function of $\phi$, the analytic map in $\mathbb{H}$ associated with $\varphi$. For a unified approach to Koenigs map for parabolic iteration, we refer the reader to [6].

We end this section by showing a technical lemma.

Lemma 3.5. Let $\phi \in \operatorname{Hol}(\mathbb{H} ; \mathbb{H})$ be parabolic with $\infty$ as Denjoy-Wolff point and denote $p(w):=\phi(w)-w, w \in \mathbb{H}$. For any two points $w, w^{*} \in \mathbb{H}$, the following inequality holds:

$$
\left|p(w)-p\left(w^{*}\right)\right| \leq 2 \frac{\widetilde{\rho}_{\mathbb{H}}\left(w, w^{*}\right)}{1-\widetilde{\rho}_{\mathbb{H}}\left(w, w^{*}\right)} \min \left\{\operatorname{Re} p(w), \operatorname{Re} p\left(w^{*}\right)\right\} .
$$

Moreover, given an arbitrary forward orbit $\left(w_{n}\right)$ of $\phi$ there exists a constant $C>0$ (not depending on $n$ ) such that, for every $n \in \mathbb{N}$ and every $w \in\left[w_{n}, w_{n+1}\right]$, we have

$$
\left|p(w)-p\left(w_{n}\right)\right| \leq C \operatorname{Re} p\left(w_{n}\right) .
$$

Proof. Since $\phi \in \operatorname{Hol}(\mathbb{H} ; \mathbb{H})$, we have that $\operatorname{Re} p(w)>0$, for all $w \in \mathbb{H}$, or $p$ is constantly equal to $i \alpha$, for some $\alpha \in \mathbb{R}$. In this second case, the lemma is trivial, so we assume that $p \in \operatorname{Hol}(\mathbb{H} ; \mathbb{H})$. Then, by the Schwarz-Pick Lemma, we obtain that for any pair of points $w, w^{*} \in \mathbb{H}$,

$$
\begin{aligned}
\left|p(w)-p\left(w^{*}\right)\right| & \leq\left|\frac{w-w^{*}}{w+\overline{w^{*}}}\right|\left|p(w)+\overline{p\left(w^{*}\right)}\right| \\
& \leq\left|\frac{w-w^{*}}{w+\overline{w^{*}}}\right|\left(2 \operatorname{Re} p\left(w^{*}\right)+\left|p(w)-p\left(w^{*}\right)\right|\right) .
\end{aligned}
$$

Hence,

$$
\left|p(w)-p\left(w^{*}\right)\right| \leq \frac{2 \widetilde{\rho}_{\mathbb{H}}\left(w, w^{*}\right)}{1-\widetilde{\rho}_{\mathbb{H}}\left(w, w^{*}\right)} \operatorname{Re} p\left(w^{*}\right)
$$

Now, take an arbitrary forward orbit $\left(w_{n}\right)$ of $\phi$. At this point, we recall the following fact on pseudo-hyperbolic distances on the right half-plane: If $a, b \in \mathbb{H}$, then

$$
\max \left\{\widetilde{\rho}_{\mathbb{H}}(w, a), \widetilde{\rho}_{\mathbb{H}}(w, b)\right\} \leq \widetilde{\rho}_{\mathbb{H}}(a, b)
$$

for every $w$ belonging to the segment $[a, b]$. Now, applying this fact to the different couples $\left\{w_{n}, w_{n+1}\right\}$, we deduce that, for every $n$ and for every $w$ belonging to the segment $\left[w_{n}, w_{n+1}\right]$,

$$
\left|p(w)-p\left(w_{n}\right)\right| \leq \frac{2 \widetilde{\rho}_{\mathbb{H}}\left(w_{n}, w_{n+1}\right)}{1-\widetilde{\rho}_{\mathbb{H}}\left(w_{n}, w_{n+1}\right)} \operatorname{Re} p\left(w_{n}\right) .
$$


Moreover, we know that $\lim _{n} \widetilde{\rho}_{\mathbb{H}}\left(w_{n}, w_{n+1}\right)$ always exists and belongs to $[0,1)$. Therefore, we can find $C>0$ which depends on the chosen orbit but not on $n$, such that

$$
\sup _{n} \frac{2 \widetilde{\rho}_{\mathbb{H}}\left(w_{n}, w_{n+1}\right)}{1-\widetilde{\rho}_{\mathbb{H}}\left(w_{n}, w_{n+1}\right)} \leq C
$$

\section{Second order Angular Differentiability: The Positive step Case}

Throughout this section, we only deal with parabolic iteration of positive h-step. In our first result, we study the relationship amongst being of angular-class of order two, being of finite shift type and the angular differentiability of Koenigs functions.

Theorem 4.1. Let $\varphi \in \operatorname{Hol}(\mathbb{D} ; \mathbb{D})$ be parabolic of positive hyperbolic step and let $\tau \in \partial \mathbb{D}$ be the corresponding Denjoy-Wolff point. The following are equivalent.

(1) The map $\varphi$ belongs to $C_{A}^{2}(\tau)$.

(2) The map $\varphi$ is of finite shift type.

(3) The map $h_{\mathbb{D}}$ belongs to $C_{A}^{1}(\tau)$, where $h_{\mathbb{D}}$ is the Koenigs function associated with $\varphi$.

Proof. As usual, we will mainly work in the right half-plane $\mathbb{H}$. Moreover, given a forward orbit $\left(z_{n}\right)$ of $\varphi$, the sequence $\left(w_{n}\right)$ will be the corresponding forward orbit of $\phi$ and vice versa. That is, $\sigma_{\tau}\left(z_{n}\right)=w_{n}$. Finally, $h$ will be the corresponding Koenigs map of $\phi$. We recall that $h \circ \phi=h+i \delta$, for some real number $\delta \neq 0$.

$(1) \Rightarrow(2)$ Suppose that $(2)$ is false and take an arbitrary orbit $\left(w_{n}\right):=\left(\phi_{n}\left(w_{0}\right)\right)$ of $\phi$ such that $\lim _{n} \operatorname{Re} w_{n}=+\infty$. Since $\varphi \in C_{A}^{2}(\tau)$ and, according to Proposition 2.1. we can assure that

$$
w_{n+1}=w_{n}+a+\gamma\left(w_{n}\right),
$$

where $a=\varphi^{\prime \prime}(\tau) \tau \in \mathbb{C}$ and $\gamma \in \operatorname{Hol}(\mathbb{H} ; \mathbb{C})$. Note that we cannot give any information about $\lim _{n} \gamma\left(w_{n}\right)$, since we do not know if $\left(w_{n}\right)$ tends non-tangentially to $\infty$.

Now, let us set $p:=a+\gamma \in \operatorname{Hol}(\mathbb{H} ; \mathbb{C})$. As usual, there are two possibilities: $\operatorname{Re} p(w)>0$, for all $w \in \mathbb{H}$, or $p$ is constantly equal to $i \alpha$, for some $\alpha \in \mathbb{R}$. In this second case, the implication is clear, so we assume that $p \in \operatorname{Hol}(\mathbb{H} ; \mathbb{H})$.

According to Lemma 3.5. we can find $C>0$ such that, for every $n \in \mathbb{N}$ and every $w \in\left[w_{n}, w_{n+1}\right]$ we have that

$$
\left|p(w)-p\left(w_{n}\right)\right| \leq C \operatorname{Rep}\left(w_{n}\right) .
$$

In addition, we also know that

$$
\frac{\operatorname{Re} p\left(w_{n}\right)}{\operatorname{Re} w_{n}}=\frac{\operatorname{Re} w_{n+1}}{\operatorname{Re} w_{n}}-1 \stackrel{n}{\rightarrow} 0 .
$$

So we obtain that

$$
\lim _{n \rightarrow \infty} \sup _{w \in\left[w_{n}, w_{n+1}\right]} \frac{\left|p(w)-p\left(w_{n}\right)\right|}{\operatorname{Re} w_{n}}=0 .
$$

Besides, we have (see the comments in section three about normalizations in the positive h-step case)

$$
\lim _{n} \frac{\operatorname{Im} p\left(w_{n}\right)}{\operatorname{Re} w_{n}}=\lim _{n} \frac{\operatorname{Im} \phi_{n}\left(\phi\left(w_{0}\right)\right)-\operatorname{Im} w_{n}}{\operatorname{Re} w_{n}}=\frac{\delta}{\operatorname{Re} h\left(w_{0}\right)} \neq 0 .
$$


Therefore,

$$
\lim _{n \rightarrow \infty} \inf _{w \in\left[w_{n}, w_{n+1}\right]}\left(\frac{\left|\operatorname{Im} p\left(w_{n}\right)\right|-\left|p(w)-p\left(w_{n}\right)\right|}{\operatorname{Re} w_{n}}\right)=\frac{|\delta|}{\operatorname{Reh}\left(w_{0}\right)}>0 .
$$

Bearing in mind the inequality

$$
|p(w)| \geq\left|\operatorname{Im} p\left(w_{n}\right)\right|-\left|p(w)-p\left(w_{n}\right)\right|=\operatorname{Re} w_{n}\left(\frac{\left|\operatorname{Im} p\left(w_{n}\right)\right|-\left|p(w)-p\left(w_{n}\right)\right|}{\operatorname{Re} w_{n}}\right),
$$

and using that $\operatorname{Re} w_{n} \rightarrow+\infty$, we conclude that

$$
\lim _{n \rightarrow \infty} \inf _{w \in\left[w_{n}, w_{n+1}\right]}|p(w)|=+\infty .
$$

Now, let us consider the polygon

$$
\Gamma:=\left[w_{0}, w_{1}\right]+\left[w_{1}, w_{2}\right]+\left[w_{2}, w_{3}\right]+\cdots .
$$

Since $p \in \operatorname{Hol}(\mathbb{H} ; \mathbb{H})$, we have that $\operatorname{Re} w_{n+1}>\operatorname{Re} w_{n}$, so $\Gamma$ is clearly a Jordan arc contained in $\mathbb{H}$ and with the value $\infty$ as the only $\omega$-limit. Thus, we have just shown that $\lim _{w \rightarrow \infty, w \in \Gamma} p(w)=\infty$ and, by Lindelöf's Principle, we deduce that

$$
\angle \lim _{w \rightarrow \infty} p(w)=\infty .
$$

But, by hypothesis and Proposition 2.1 , we know that the $\operatorname{limit} \angle \lim _{w \rightarrow \infty} p(w)$ exists finitely, so we have a contradiction.

$(2) \Rightarrow(1)$ Fix an orbit $\left(w_{n}\right):=\left(\phi_{n}\left(w_{0}\right)\right)$ of $\phi$ and denote $\beta=\beta\left(w_{0}\right):=$ $\lim _{n} \operatorname{Re} w_{n} \in(0,+\infty)$. In addition, let us set $p(w):=\phi(w)-w, w \in \mathbb{H}$. Once again, we have two possibilities: $(i) p(w)=i \alpha$, for some non-zero $\alpha \in \mathbb{R}$; (ii) $p \in \operatorname{Hol}(\mathbb{H} ; \mathbb{H})$.

In the first case, $\phi$ is an automorphism of $\mathbb{H}$ and so is $\varphi$ in $\mathbb{D}$. But, automorphisms in the unit disk are analytic beyond $\overline{\mathbb{D}}$. Therefore, $\varphi \in C_{A}^{2}(b)$ for any boundary fixed point of $\varphi$.

The second case requires more work. Using [9], we find

$$
\lim _{n} \operatorname{Re} p\left(w_{n}\right)=\lim _{n}\left(\operatorname{Re} w_{n+1}-\operatorname{Re} w_{n}\right)=\lim _{n} \operatorname{Re} w_{n}\left(\frac{\operatorname{Re} w_{n+1}}{\operatorname{Re} w_{n}}-1\right)=\beta \cdot 0=0 .
$$

Again using Lemma 3.5, we find $C>0$ such that, for every $n \in \mathbb{N}$ and every $w \in\left[w_{n}, w_{n+1}\right]$ we have that

$$
\left|p(w)-p\left(w_{n}\right)\right| \leq C \operatorname{Re} p\left(w_{n}\right) .
$$

Therefore,

$$
L:=\lim _{n \rightarrow \infty} \sup _{w \in\left[w_{n}, w_{n+1}\right]}\left|p(w)-p\left(w_{n}\right)\right| \leq C \lim _{n \rightarrow \infty} \operatorname{Re} p\left(w_{n}\right)=0 .
$$

Now, let us consider the polygon

$$
\Gamma:=\left[w_{0}, w_{1}\right]+\left[w_{1}, w_{2}\right]+\left[w_{2}, w_{3}\right]+\cdots .
$$

Since $p \in \operatorname{Hol}(\mathbb{H} ; \mathbb{H})$, we have again that $\operatorname{Re} w_{n+1}>\operatorname{Re} w_{n}$, and $\Gamma$ is a Jordan arc contained in $\mathbb{H}$ with the value $\infty$ as the only $\omega$-limit. Then, using that $L=0$, we 
find that

$$
\begin{aligned}
\lim _{w \rightarrow \infty, w \in \Gamma} p(w) & =\lim _{n \rightarrow \infty} p\left(w_{n}\right) \\
& =\lim _{n \rightarrow \infty} \operatorname{Re} w_{n}\left(\frac{\operatorname{Re} w_{n+1}-\operatorname{Re} w_{n}}{\operatorname{Re} w_{n}}+i \frac{\operatorname{Im} w_{n+1}-\operatorname{Im} w_{n}}{\operatorname{Re} w_{n}}\right) \\
& =i \frac{\beta \delta}{\operatorname{Reh}\left(w_{0}\right)} .
\end{aligned}
$$

Again, by Lindelöf's Principle, we deduce that

$$
\angle \lim _{w \rightarrow \infty} p(w)=i \frac{\beta \delta}{\operatorname{Re} h\left(w_{0}\right)} .
$$

Finally, appealing to Proposition 2.1, we have that $\varphi \in C_{A}^{2}(\tau)$ and, indeed,

$$
\varphi^{\prime \prime}(\tau)=i \frac{\bar{\tau} \beta \delta}{\operatorname{Reh}\left(w_{0}\right)} .
$$

$(2) \Rightarrow(3)$ Consider the specific orbit $\left(w_{n}\right):=\left(\phi_{n}(1)\right)$ and denote $x_{n}=\operatorname{Re} w_{n}$, $y_{n}=\operatorname{Im} w_{n}$. By hypothesis, we have that $\beta=\beta(1):=\lim _{n} x_{n} \in(0,+\infty)$. Recall that $h \in \operatorname{Hol}(\mathbb{H} ; \mathbb{H})$; so, bearing in mind the Julia-Carathéodory Theorem, we see that $h_{\mathbb{D}} \in C_{A}^{1}(\tau)$ if and only if

$$
h^{\prime}(\infty)=\inf \left\{\frac{\operatorname{Re} h(w)}{\operatorname{Re} w}: w \in \mathbb{H}\right\}>0 .
$$

We note that all the iterates $\phi_{n}$ are parabolic. Therefore, for any $w \in \mathbb{H}$,

$$
\frac{1}{\operatorname{Re} w} \operatorname{Re}\left(\frac{\phi_{n}(w)-i y_{n}}{x_{n}}\right)=\frac{\operatorname{Re} \phi_{n}(w)}{x_{n} \operatorname{Re} w} \geq \frac{1}{x_{n}} \geq \frac{1}{\beta} .
$$

Taking limits in $n$ (with $w$ fixed), we obtain

$$
\frac{\operatorname{Reh}(w)}{\operatorname{Re} w} \geq \frac{1}{\beta}>0
$$

as wanted. Moreover, $\beta h^{\prime}(\infty)>1$.

$(3) \Rightarrow(2)$ Since being of finite shift type does not depend on the chosen orbit, we consider again the specific orbit $\left(w_{n}\right):=\left(\phi_{n}(1)\right)$ and suppose that $\operatorname{Re} w_{n} \rightarrow+\infty$. Since

we have

$$
h\left(w_{n}\right)=h(1)+i n \delta, \text { for } n \in \mathbb{N},
$$

$$
\frac{\operatorname{Re} h\left(w_{n}\right)}{\operatorname{Re} w_{n}}=\frac{h(1)}{\operatorname{Re} w_{n}} \stackrel{n}{\rightarrow} 0 .
$$

Therefore, $h^{\prime}(\infty)=\inf \left\{\frac{\operatorname{Re} h(w)}{\operatorname{Re} w}: w \in \mathbb{H}\right\}=0$ and the corresponding $h_{\mathbb{D}}:=h \circ$ $\sigma_{\tau} \notin C_{A}^{1}(\tau)$.

Looking carefully at the proof of the above theorem, we discover the following dichotomy.

Corollary 4.2. Assume that $\varphi \in \operatorname{Hol}(\mathbb{D} ; \mathbb{D})$ is a parabolic map of positive hyperbolic step with Denjoy-Wolff point $\tau \in \partial \mathbb{D}$. Then either

$$
\angle \lim _{z \rightarrow \tau} \frac{\varphi(z)-z}{(\tau-z)^{2}}=\infty
$$

or $\varphi \in C_{A}^{2}(\tau)$ and, in this second case, $\operatorname{Re}\left(\tau \varphi^{\prime \prime}(\tau)\right)=0$. 
For our next result, we must say some words about natural procedures to obtain intertwining maps. Given a hyperbolic map $\phi \in \operatorname{Hol}(\mathbb{D} ; \mathbb{D})$ with $\infty$ as Denjoy-Wolff point and $c=\phi^{\prime}(\infty)>1$, we can consider the normalized sequence

$$
h_{n}(w):=\frac{\phi_{n}(w)}{c^{n}}, w \in \mathbb{H}
$$

and study its convergence. For many reasons (going back to the works of Koenigs, Wolff and Valiron), this normalization can be named "the natural one" and when it converges non-trivially it produces a certain intertwining map. It is a remarkable fact that this natural normalization does not always work, so it is interesting to ask when it does. This problem has already been treated by Wolff and Valiron and more recently by the third author [10] who proved that $\left(h_{n}\right)$ converges non-trivially in the compact open topology of $\mathbb{H}$ if and only if the complex line integral

$$
\int_{1}^{+\infty} \frac{|\phi(t)-c t|}{t^{2}} d t=\int_{\gamma} \frac{|\phi(z)-c z|}{z^{2}} d z \quad(\gamma(t)=t, t \geq 1)
$$

converges.

Our next theorem says that being of angular-class two at the Denjoy-Wolff point is equivalent to what could be considered the natural normalization in the parabolic case. We also provide a characterization involving a line integral quite similar in spirit to the one given in [10] for the hyperbolic case. The main difference now is that we impose a tangential approach to infinity on our corresponding curve.

Theorem 4.3. Let $\varphi \in \operatorname{Hol}(\mathbb{D} ; \mathbb{D})$ be parabolic of positive hyperbolic step with Denjoy-Wolff point $\tau \in \partial \mathbb{D}$. Likewise, let $\phi$ be the associated map with $\varphi$ in $\mathbb{H}$. Then the following are equivalent.

(1) $\varphi$ belongs to $C_{A}^{2}(\tau)$.

(2) For some (resp. any) $w_{0} \in \mathbb{H}$, the normalized sequence

$$
g_{n}(w):=\phi_{n}(w)-\phi_{n}\left(w_{0}\right), w \in \mathbb{H}
$$

converges in the compact open topology to some non-constant $g \in \operatorname{Hol}(\mathbb{H} ; \mathbb{C})$ (of course, $g$ depends on $w_{0}$ ).

(3) The complex line integral

$$
\int_{\gamma} \operatorname{Re}(\phi(z)-z) d z
$$

converges, where

$$
\gamma(t)=\left\{\begin{array}{l}
1+i t, \text { if } \phi \text { is of type } I, \\
1-i t, \text { if } \phi \text { is of type II, }
\end{array} \quad t \geq 1 .\right.
$$

If any of the above statements holds, then there exists a real number $\nu \neq 0$, such that $g \circ \phi=g+i \nu$.

Proof. As usual, $h$ will be the corresponding Koenigs map of $\phi, x_{n}=\operatorname{Re} \phi_{n}(1)$ and $y_{n}=\operatorname{Im} \phi_{n}(1)$. We recall that $h \in \operatorname{Hol}(\mathbb{H} ; \mathbb{H})$ and $h \circ \phi=h+i \delta$, for some $\delta \neq 0$. We also write $p(w)=\phi(w)-w, w \in \mathbb{H}$.

$(1) \Rightarrow(2)$ Take an arbitrary $w_{0} \in \mathbb{H}$ and denote $w_{n}=\phi_{n}\left(w_{0}\right)$. According to Theorem 4.1. we know that $\operatorname{Re} \phi_{n}\left(w_{0}\right) \rightarrow \beta \in(0,+\infty)$ and $x_{n} \rightarrow \mu \in(0,+\infty)$. 
Moreover, for any $w \in \mathbb{H}$,

$$
\begin{aligned}
\phi_{n}(w)-\phi_{n}\left(w_{0}\right)= & x_{n} \frac{\phi_{n}(w)-i y_{n}}{x_{n}}-x_{n} i \frac{\operatorname{Im} \phi_{n}\left(w_{0}\right)-y_{n}}{x_{n}}-\operatorname{Re} \phi_{n}\left(w_{0}\right) \\
& \stackrel{n}{\longrightarrow} \mu h(w)-i \mu \operatorname{Im} h\left(w_{0}\right)-\beta .
\end{aligned}
$$

Since the above convergence to $h$ is locally uniformly in $\mathbb{H}$, the implication is proved and, indeed, $g=\mu h-\left(\beta+i \mu \operatorname{Im} h\left(w_{0}\right)\right)$ and $g \circ \phi=g+i \delta \mu$.

$(2) \Rightarrow(1)$ Fix $w_{0} \in \mathbb{H}$, denote $w_{n}=\phi_{n}\left(w_{0}\right)$ and take the corresponding $g$. Our hypothesis guarantees the existence of the limit

$$
\beta:=\lim _{n}\left(\operatorname{Re} w_{n+1}-\operatorname{Re} w_{n}\right)=\lim _{n} \operatorname{Re} p\left(w_{n}\right) .
$$

Since the sequence $\left(\operatorname{Re} w_{n}\right)$ is non-decreasing, we have $\beta \in[0,+\infty)$. Let us check that $\beta=0$. Otherwise, using (2), we deduce (see again the comments about normalizations in the positive h-step case)

$$
\begin{aligned}
0 & \neq \frac{\delta}{\operatorname{Re} h\left(w_{0}\right)}=\operatorname{Im}\left(\frac{h\left(\phi\left(w_{0}\right)\right)-i \operatorname{Im} h\left(w_{0}\right)}{\operatorname{Re} h\left(w_{0}\right)}\right)=\lim _{n} \frac{\operatorname{Im} w_{n+1}-\operatorname{Im} w_{n}}{\operatorname{Re} w_{n}} \\
& =\lim _{n} \frac{\operatorname{Im} \phi_{n}\left(\phi\left(w_{0}\right)\right)-\operatorname{Im} \phi_{n}\left(w_{0}\right)}{\operatorname{Re} w_{n+1}-\operatorname{Re} w_{n}}\left(\frac{\operatorname{Re} w_{n+1}}{\operatorname{Re} w_{n}}-1\right) \\
& =\frac{\operatorname{Im} g\left(\phi\left(w_{0}\right)\right)}{\beta}(1-1)=0 .
\end{aligned}
$$

Hence, we have a contradiction unless $\beta=0$. But, again according to Lemma 3.5. this implies that

$$
\lim _{n \rightarrow \infty} \sup _{w \in\left[w_{n}, w_{n+1}\right]}\left|p(w)-p\left(w_{n}\right)\right|=0 .
$$

Once again, we consider the Jordan $\operatorname{arc} \Gamma=\left[w_{0}, w_{1}\right]+\left[w_{1}, w_{2}\right]+\left[w_{2}, w_{3}\right]+\cdots$, and following the same kind of arguments as in $(2) \Rightarrow(1)$ of Theorem 4.1, we deduce that

$$
\lim _{w \rightarrow \infty, w \in \Gamma} p(w)=\lim _{n \rightarrow \infty} p\left(w_{n}\right)=\lim _{n \rightarrow \infty}\left(\phi_{n}\left(\phi\left(w_{0}\right)\right)-\phi_{n}\left(w_{0}\right)\right)=g\left(\phi\left(w_{0}\right)\right) .
$$

Therefore, we conclude that $\varphi \in C_{A}^{2}(\tau)$ and, indeed, $\varphi^{\prime \prime}(\tau)=\bar{\tau} g\left(\phi\left(w_{0}\right)\right)$.

$(1) \Rightarrow(3)$ We only prove the type I case (the other is completely similar). In this case, the above $\delta$ is positive. Moreover, since $h(1)=1$ and

$$
0<\delta=\lim _{n} \frac{y_{n+1}-y_{n}}{x_{n}},
$$

we deduce that there exists $N \in \mathbb{N}$ such that $\left(y_{n}\right)_{n \geq N}$ is a strictly increasing sequence of real numbers and there exists $C_{1}>0$ such that

$$
y_{n+1}-y_{n} \leq C_{1} x_{n}, \text { for every } n \geq N .
$$

We claim that there exists another constant $C_{2} \in(0,1)$ such that for every $n \in \mathbb{N}$ and for every $w$ belonging to the segment $\left[1+i y_{n}, 1+i y_{n+1}\right]$, we have that

$$
\widetilde{\rho}_{\mathbb{H}}\left(w, \phi_{n}(1)\right) \leq C_{2} .
$$

We may assume that $p \in \operatorname{Hol}(\mathbb{H} ; \mathbb{H})$ since the result is trivial when $p=i \alpha$, $\alpha \in \mathbb{R}$. Moreover, using Lemma 3.5, we obtain that for every $n \in \mathbb{N}$ and for every 
$w \in\left[1+i y_{n}, 1+i y_{n+1}\right]$

$$
\left|\operatorname{Re} p(w)-\operatorname{Re} p\left(\phi_{n}(1)\right)\right| \leq 2 \frac{\widetilde{\rho}_{\mathbb{H}}\left(w, \phi_{n}(1)\right)}{1-\widetilde{\rho}_{\mathbb{H}}\left(w, \phi_{n}(1)\right)} \operatorname{Re} p\left(\phi_{n}(1)\right) \leq \frac{2 C_{2}}{1-C_{2}}\left(x_{n+1}-x_{n}\right) .
$$

Note that $C_{3}:=\frac{2 C_{2}}{1-C_{2}}>0$ since $C_{2}<1$.

Bearing in mind our hypothesis and Theorem 4.1, we know that there exists $\beta=\sup _{n} x_{n}=\lim _{n} x_{n} \in(0,+\infty)$ and $y_{n} \rightarrow+\infty$. Hence,

$$
\begin{aligned}
\int_{y_{N}}^{+\infty} \operatorname{Rep}(1+i t) d t & \leq \sum_{n=N}^{\infty} \int_{y_{n}}^{y_{n+1}}\left(\left|\operatorname{Re} p(1+i t)-\operatorname{Re} p\left(\phi_{n}(1)\right)\right|+\operatorname{Rep}\left(\phi_{n}(1)\right)\right) d t \\
& \leq\left(C_{3}+1\right) \sum_{n=N}^{\infty}\left(x_{n+1}-x_{n}\right)\left(y_{n+1}-y_{n}\right) \\
& \leq\left(C_{3}+1\right) C_{1} \sum_{n=N}^{\infty}\left(x_{n+1}-x_{n}\right) x_{n} \\
& \leq\left(C_{3}+1\right) C_{1} \beta\left(\beta-x_{N}\right)<+\infty
\end{aligned}
$$

This clearly proves that the integral converges.

Proof of the claim. Again, set $\beta=\lim _{n} x_{n} \in(0,+\infty)$ and suppose that the claim is false. Then, we can find a convergent sequence $\left(\lambda_{k}\right)$ in $[0,1]$ and a subsequence $\left(\phi_{n_{k}}(1)\right)$ such that

$$
\lim _{k} \widetilde{\rho}_{\mathbb{H}}\left(1+i \lambda_{k} y_{n_{k}}+i\left(1-\lambda_{k}\right) y_{n_{k}+1}, \phi_{n_{k}}(1)\right)=1 .
$$

However, writing $\lambda=\lim _{k} \lambda_{k} \in[0,1]$ and recalling that $\delta=\lim _{n} \frac{y_{n+1}-y_{n}}{x_{n}}$ we find that

$$
\lim _{k} \widetilde{\rho}_{\mathbb{H}}\left(1+i \lambda_{k} y_{n_{k}}+i\left(1-\lambda_{k}\right) y_{n_{k}+1}, \phi_{n_{k}}(1)\right)=\left|\frac{1-\frac{1}{\beta}-i(1-\lambda) \delta}{1+\frac{1}{\beta}+i(1-\lambda) \delta}\right|<1 .
$$

$(3) \Rightarrow(1)$ As above, we will only prove the implication for the type I case, that is, when $\delta>0$. According to Theorem 4.1, it is enough to prove that the sequence $\left(x_{n}\right)$ is convergent. We assume that $p \in \operatorname{Hol}(\mathbb{H} ; \mathbb{H}$ ) (when $p$ is constant, the implication is trivial by Proposition 2.1).

Using the Schwarz-Pick Lemma (see the formula (3.2) in [9]), we find that, for every $v \in \mathbb{R}$, the corresponding function

$$
t \in[0,+\infty) \longrightarrow \frac{\operatorname{Re} p(t+i v)}{t} \in[0,+\infty)
$$

is non-increasing. Since $x_{n}=\operatorname{Re} \phi_{n}(1) \geq \operatorname{Re} \phi(1) \geq 1$, we deduce that, for every $n \in \mathbb{N}$ and every $v \in \mathbb{R}$,

$$
\frac{\operatorname{Re} p\left(x_{n}+i v\right)}{x_{n}} \leq \operatorname{Rep}(1+i v) .
$$

Now we use the same fact considered in the proof of Lemma 3.5. Namely, if $a, b \in \mathbb{H}$, then

$$
\max \left\{\widetilde{\rho}_{\mathbb{H}}(w, a), \widetilde{\rho}_{\mathbb{H}}(w, b)\right\} \leq \widetilde{\rho}_{\mathbb{H}}(a, b)
$$


for every $w$ belonging to the segment $[a, b]$. Therefore, for every $n \in \mathbb{N}$ and for every $w$ belonging to the segment $\left[x_{n}+i y_{n}, x_{n}+i y_{n+1}\right]$, we have that

$$
\widetilde{\rho}_{\mathbb{H}}\left(w, \phi_{n}(1)\right) \leq \widetilde{\rho}_{\mathbb{H}}\left(x_{n}+i y_{n+1}, x_{n}+i y_{n}\right)=\frac{\left|\frac{y_{n+1}-y_{n}}{x_{n}}\right|}{\left|2+\frac{y_{n+1}-y_{n}}{x_{n}} i\right|} .
$$

Since $\delta=\lim _{n} \frac{y_{n+1}-y_{n}}{x_{n}} \neq 0$, we conclude that there exists a constant $C \in(0,1)$ such that for every $n \in \mathbb{N}$ and for every $w$ belonging to the segment $\left[x_{n}+i y_{n}, x_{n}+\right.$ $\left.i y_{n+1}\right]$, we have

$$
\widetilde{\rho}_{\mathbb{H}}\left(w, \phi_{n}(1)\right) \leq C .
$$

Then, again using Lemma 3.5 and this uniform bound, we find that for every $n \in \mathbb{N}$ and for every $w \in\left[x_{n}+i y_{n}, x_{n}+i y_{n+1}\right]$, we have that

$$
\operatorname{Re} p\left(\phi_{n}(1)\right) \leq \operatorname{Re} p(w)+\left|p(w)-p\left(\phi_{n}(1)\right)\right| \leq \frac{1+C}{1-C} \operatorname{Re} p(w) .
$$

Now, as in the beginning of the implication $(1) \Rightarrow(3)$, we can also obtain $N \in \mathbb{N}$ and a constant $C_{1}>0$ such that, for every $n \geq N$,

$$
0<\frac{1}{C_{1}} \leq \frac{y_{n+1}-y_{n}}{x_{n}} \leq C_{1} .
$$

Moreover, for a fixed $n \geq N$ and bearing in mind (*), we compute

$$
\begin{aligned}
\int_{y_{n}}^{y_{n+1}} \operatorname{Re} p(1+i t) d t & \geq \frac{1}{x_{n}} \int_{y_{n}}^{y_{n+1}} \operatorname{Re} p\left(x_{n}+i t\right) d t \\
& \geq \frac{1-C}{x_{n}(1+C)} \int_{y_{n}}^{y_{n+1}} \operatorname{Re} p\left(\phi_{n}(1)\right) d t \\
& =\frac{1-C}{1+C} \operatorname{Re} p\left(\phi_{n}(1) \frac{y_{n+1}-y_{n}}{x_{n}} \geq \frac{1-C}{(1+C) C_{1}} \operatorname{Re} p\left(\phi_{n}(1)\right) .\right.
\end{aligned}
$$

Therefore, using (3), we deduce that

$$
\begin{aligned}
\sum_{n=N}^{\infty}\left|x_{n+1}-x_{n}\right| & =\sum_{n=N}^{\infty}\left(x_{n+1}-x_{n}\right)=\sum_{n=N}^{\infty} \operatorname{Re} p\left(\phi_{n}(1)\right) \\
& \leq \frac{C_{1}(1+C)}{1-C} \sum_{n=N}^{\infty} \int_{y_{n}}^{y_{n+1}} \operatorname{Re} p(1+i t) d t \\
& =\frac{C_{1}(1+C)}{1-C} \int_{y_{N}}^{\infty} \operatorname{Re} p(1+i t) d t<+\infty
\end{aligned}
$$

Since this series is telescopic, we conclude that the sequence $\left(x_{n}\right)$ is convergent as wanted.

Remark. As one might expect, the line integral condition of the former theorem can be translated to a formula in the unit disk. Namely, assume that $\varphi \in \operatorname{Hol}(\mathbb{D} ; \mathbb{D})$ is parabolic of positive h-step and with Denjoy-Wolff point $\tau \in \partial \mathbb{D}$. Then, it is possible to prove that $\varphi \in C_{A}^{2}(\tau)$ if and only if the complex line integral

$$
\int_{\gamma}\left(\frac{1-|\varphi(z)|^{2}}{|\tau-\varphi(z)|^{2}}-1\right) \frac{|d z|}{1-|z|^{2}}
$$


is convergent, where

$$
\gamma(t)=\left\{\begin{array}{l}
\frac{i t}{2+i t}, \text { if } \varphi \text { is of type I, } \\
\frac{-i t}{2-i t}, \text { if } \varphi \text { is of type II, }
\end{array} \quad t \geq 0 .\right.
$$

We end this section by giving an orbit estimation. Essentially, we prove that any orbit tends $n$-linearly to the Denjoy-Wolff point under the hypothesis of second angular differentiability at this point.

Proposition 4.4. Let $\varphi \in \operatorname{Hol}(\mathbb{D} ; \mathbb{D})$ be parabolic of positive hyperbolic step with Denjoy-Wolff point $\tau \in \partial \mathbb{D}$. Likewise, let $\phi$ be the analytic map in $\mathbb{H}$ associated with $\varphi$ and assume that $\varphi$ belongs to $C_{A}^{2}(\tau)$. Then there exists a non-zero real number $b$ such that

$$
\lim _{n \rightarrow \infty} \frac{\phi_{n}(w)}{n}=i b
$$

uniformly on compact subsets of $\mathbb{H}$.

Proof. We consider again the corresponding Koenigs map $h$ (normalized with respect to 1) associated with $\phi$ and recall that $h \in \operatorname{Hol}(\mathbb{H} ; \mathbb{H})$ and $h \circ \phi=h+i \delta$, for some $\delta \neq 0$. We also write $x_{n}=\operatorname{Re} \phi_{n}(1)$ and $y_{n}=\operatorname{Im} \phi_{n}(1)$.

By Theorem 4.1, we know that $\lim _{n} x_{n}=\beta \in(0,+\infty)$. Therefore,

$$
y_{n+1}-y_{n}=x_{n} \frac{y_{n+1}-y_{n}}{x_{n}} \stackrel{n}{\rightarrow} \beta \delta .
$$

Therefore, applying the Cesaro-criterion limit, we also deduce that

$$
\lim _{n \rightarrow \infty} \frac{y_{n}}{n}=\lim _{n \rightarrow \infty} \frac{y_{n}-y_{0}}{n}=\lim _{n \rightarrow \infty} \frac{1}{n} \sum_{k=0}^{n-1}\left(y_{k+1}-y_{k}\right)=\beta \delta .
$$

Bearing in mind Theorem 4.3 we take the corresponding map $g \in \operatorname{Hol}(\mathbb{D} ; \mathbb{C})$ and the real number $\nu \neq 0$ related to the point 1 . In fact, looking at the proof, we see that $\nu=\beta \delta$. Therefore,

$$
\lim _{n \rightarrow \infty} \frac{\phi_{n}(w)}{n}=\lim _{n \rightarrow \infty} \frac{1}{n}\left(\phi_{n}(w)-\phi_{n}(1)\right)+i \lim _{n \rightarrow \infty} \frac{y_{n}}{n}=\beta \delta i .
$$

Clearly, this convergence holds uniformly on compact subsets of $\mathbb{H}$.

\section{General Results for parabolic functions}

From the technical point of view, there is a big difference between dealing with the zero step case instead of the positive one. Now, in general, the Koenigs map does not leave $\mathbb{H}$ invariant, so we cannot use the Schwarz-Pick Lemma and related results. We need new tools. The next theorem is one of them and describes certain subsets that are relatively easy to handle where the Koenigs map is univalent.

Following Cowen, a domain $V$ in $\mathbb{D}$ is called a fundamental set for $\varphi \in \operatorname{Hol}(\mathbb{D} ; \mathbb{D})$ if it is a non-void, open, connected and simply connected set such that $\varphi(V) \subset V$ and, for every compact $K \subset \mathbb{D}$, there exists a positive integer $N$ such that $\varphi_{N}(K) \subset V$. We recall that Cowen [5] proved the existence of a fundamental set for all types of $\varphi$. The next theorem contains an explicit construction restricted to the zero hyperbolic step case. Note that statements (1) to (4) are for general parabolic iteration. 
Theorem 5.1. Let $\varphi \in \operatorname{Hol}(\mathbb{D} ; \mathbb{D})$ be parabolic and $\tau \in \partial \mathbb{D}$ the corresponding Denjoy-Wolff point. Given $0<\varrho<1$, write

$$
V(\varrho)=\left\{z \in \mathbb{D}: \widetilde{\rho}_{\mathbb{D}}(z, \varphi(z))<\varrho\right\} .
$$

Then,

(1) $\varphi(V(\varrho)) \subset V(\varrho)$ for all $0<\varrho<1$.

(2) $V(\varrho)$ is a simply connected domain.

(3) Given a Stolz angle $S(\tau, \alpha)=\{z \in \mathbb{D}:|\operatorname{Arg}(1-\bar{\tau} z)|<\alpha\}$ and $0<\varrho<1$, there is $\rho>0$ such that $S(\tau, \alpha) \cap\{z \in \mathbb{D}:|\tau-z|<\rho\}$ is a subset of $V(\varrho)$.

(4) If $0<\varrho \leq \frac{1}{3}$, then $\varphi$ and the Koenigs map are univalent in $V(\varrho)$.

(5) If $\varphi$ is of zero hyperbolic step and $0<\varrho \leq \frac{1}{3}$, then $V(\varrho)$ is a fundamental set for the function $\varphi$.

Proof. As usual, we prove the above statement for the right half-plane $\mathbb{H}$. In this sense, we denote by $\phi$ the iteration map in $\mathbb{H}$ associated with $\varphi$. Recall that $\phi \in \operatorname{Hol}(\mathbb{H} ; \mathbb{H})$ and it is parabolic with $\infty$ as Denjoy-Wolff point. As usual, we write $p(w)=\phi(w)-w$. To simplify the notation, we also write $W(\varrho)=$ $\left\{w \in \mathbb{H}: \widetilde{\rho}_{\mathbb{H}}(w, \phi(w))<\varrho\right\}$, that is, $W(\varrho)=\sigma_{\tau}(V(\varrho))$. Note that $w=u+i v \in W(\varrho)$ if and only if $\left|\frac{p(w)}{p(w)+2 u}\right|<\varrho$ and if and only if $\left|\frac{2 u}{p(w)}+1\right|>\frac{1}{\varrho}$.

Statement (1) is just a consequence of the fact that $\varphi$ is a contraction in the pseudo-hyperbolic distance. Moreover, statements (2) and (3) are easy consequences of the following two claims:

Claim 1. If $w_{0} \in W(\varrho)$, then $w_{0}+t \in W(\varrho)$ for all $t \geq 0$.

Claim 2. Given $\lambda>0$, there is $u_{0}$ such that

$$
\left\{u+i v \in \mathbb{H}: u \geq u_{0},|v| \leq \lambda u\right\} \subset W(\varrho) .
$$

Proof of Claim 1. By [9, equation (3.2)],

$$
\left|p^{\prime}(w)\right| \leq \frac{\operatorname{Re} p(w)}{\operatorname{Re} w} \leq \frac{|p(w)|}{\operatorname{Re} w}
$$

for all $w \in \mathbb{H}$. Therefore, given $w=u+i v \in \mathbb{H}$ and using the above inequalities (5.1), we have

$$
\begin{aligned}
\frac{\partial}{\partial u}\left|\frac{2 u}{p(w)}+1\right|^{2} & =\frac{\partial}{\partial u}\left(\frac{4 u^{2}}{|p(w)|^{2}}\right)+\frac{\partial}{\partial u}\left(\operatorname{Re} \frac{4 u}{p(w)}\right) \\
& =\frac{8 u|p(w)|^{2}-8 u^{2} \operatorname{Re}\left(p^{\prime}(w) \overline{p(w)}\right)}{|p(w)|^{4}}+\operatorname{Re}\left(\frac{4}{p(w)}-\frac{4 u p^{\prime}(w)}{p(w)^{2}}\right) \\
& \geq \frac{8 u}{|p(w)|^{2}}\left(\frac{|p(w)|-u\left|p^{\prime}(w)\right|}{|p(w)|}\right)+4 \frac{\operatorname{Re} p(w)-u\left|p^{\prime}(w)\right|}{|p(w)|^{2}} \geq 0
\end{aligned}
$$

Now, take $w_{0}=u+i v \in W(\varrho)$ and $t \geq 0$. Since $u \mapsto\left|\frac{2 u}{p(w)}+1\right|^{2}$ is non-decreasing in the interval $(0,+\infty)$, we obtain

$$
\left|\frac{2(u+t)}{p\left(w_{0}+t\right)}+1\right|^{2} \geq\left|\frac{2 u}{p\left(w_{0}\right)}+1\right|^{2} \geq \frac{1}{\varrho^{2}} .
$$

This proves Claim 1. 
Proof of Claim 2. Recall that $\angle \lim _{w \rightarrow \infty} \frac{p(w)}{w}=0$. Therefore, given $\lambda>0$, there is $u_{0}>0$ such that

$$
\left|\frac{p(w)}{w}\right|<\frac{2 \varrho}{\sqrt{1+\lambda^{2}}}
$$

whenever $w=u+i v,|v| \leq \lambda u$ and $u \geq u_{0}$. Moreover, since $\operatorname{Re} \frac{2 u}{p(w)}>0$ and $|w|=|u+i v| \leq \sqrt{1+\lambda^{2}} u$, we have

$$
\left|\frac{2 u}{p(w)}+1\right| \geq \frac{2 u}{|p(w)|} \geq \frac{2}{\sqrt{1+\lambda^{2}}} \frac{|w|}{|p(w)|}>\frac{1}{\varrho} .
$$

This proves Claim 2.

Now, we prove statement (4). Fix $0<\varrho \leq \frac{1}{3}$. If $w=u+i v \in W(\varrho)$, we have that $\left|\frac{2 u}{p(w)}+1\right|>\frac{1}{\varrho}$ and, therefore, $\frac{2 u}{|p(w)|}>\frac{1}{\varrho}-1 \geq 2$. That is, $\frac{|p(w)|}{\operatorname{Re} w}<1$ for all $w \in W(\varrho)$. Besides, by [9, equation (3.3)], we have

$$
\left|\frac{p\left(w_{1}\right)-p\left(w_{2}\right)}{w_{1}-w_{2}}\right|^{2} \leq \frac{\operatorname{Re} p\left(w_{1}\right)}{\operatorname{Re} w_{1}} \frac{\operatorname{Re} p\left(w_{2}\right)}{\operatorname{Re} w_{2}}
$$

for all $w_{1}, w_{2} \in \mathbb{H}$. Therefore, given $w_{1}, w_{2} \in W(\varrho), w_{1} \neq w_{2}$, we obtain that

$$
\left|\frac{p\left(w_{1}\right)-p\left(w_{2}\right)}{w_{1}-w_{2}}\right|^{2} \leq \frac{\left|p\left(w_{1}\right)\right|}{\operatorname{Re} w_{1}} \frac{\left|p\left(w_{2}\right)\right|}{\operatorname{Re} w_{2}}<1 .
$$

That is, $\left|p\left(w_{1}\right)-p\left(w_{2}\right)\right|<\left|w_{1}-w_{2}\right|$. Hence,

$$
\left|\phi\left(w_{1}\right)-\phi\left(w_{2}\right)\right| \geq\left|w_{1}-w_{2}\right|-\left|p\left(w_{1}\right)-p\left(w_{2}\right)\right|>0
$$

whenever $w_{1}, w_{2} \in W(\varrho), w_{1} \neq w_{2}$. This clearly implies that $\phi$ is univalent in $W(\varrho)$.

Now, we show that the Koenigs map is also univalent in $W(\varrho)$ for $0<\varrho \leq \frac{1}{3}$. By [9] and [1] (see also [6]), the Koenigs map is the limit in the compact-open topology of $\mathbb{H}$ of a sequence of functions

$$
h_{n}(w)=a_{n} \phi_{n}(w)+b_{n}, w \in \mathbb{H},
$$

for some complex numbers $a_{n}, b_{n} \in \mathbb{C}, a_{n} \neq 0$. We know that $\phi(W(\varrho)) \subset W(\varrho)$ and $\phi$ is univalent in $W(\varrho)$. Therefore, $\phi_{n}$, and hence $h_{n}$, is univalent in $W(\varrho)$ for all $n$. Finally, applying Hurwitz's Theorem, we deduce that the corresponding Koenigs map is univalent in $W(\varrho)$.

Finally, bearing in mind (4), to obtain (5), it is enough to show that for all $w \in \mathbb{H}$, there is $n \in \mathbb{N}$ such that $\phi_{n}(w) \in W(\varrho)$. But this is obvious since now

$$
\lim _{n \rightarrow \infty} \widetilde{\rho}_{\mathbb{H}}\left(\phi_{n}(w), \phi_{n+1}(w)\right)=0
$$

Remark. Statement (5) in the above theorem is not true, in general, for the positive h-step case, as is shown by the functions $\varphi$ obtained from $\phi(w)=w+i b$, for large positive $b$. It is also worth pointing out that the constant $1 / 3$ in statement (4) of the above theorem is sharp. Namely, let us consider the function $\varphi(z)=\frac{3 z^{2}+1}{3+z^{2}}$ for 
all $z \in \mathbb{D}$. In this case, we have that $\phi(w)=w+\frac{1}{w}$. That is, $p(w)=\frac{1}{w}$. Therefore

$$
\begin{aligned}
W(\varrho) & =\left\{w \in \mathbb{H}: \widetilde{\rho}_{\mathbb{H}}(w, \phi(w))<\varrho\right\} \\
& =\left\{w=u+i v \in \mathbb{H}: 4 u^{2}\left(u^{2}+v^{2}\right)+4 u^{2}+1>\frac{1}{\varrho^{2}}\right\} .
\end{aligned}
$$

Then, $1 \in W(\varrho)$ whenever $\varrho>\frac{1}{3}$. But $\phi^{\prime}(1)=0$ and we have that $\phi$ is not univalent in $W(\varrho)$.

Our next result describes the non-tangential angular asymptotic behaviour of the Koenigs map and will be a major tool for our study of second angular differentiability.

Theorem 5.2. Let $\varphi \in \operatorname{Hol}(\mathbb{D} ; \mathbb{D})$ be parabolic and let $\tau \in \partial \mathbb{D}$ be the corresponding Denjoy-Wolff point. Then

$$
\angle \lim _{z \rightarrow \tau}(\varphi(z)-z) h_{\mathbb{D}}^{\prime}(z)=1
$$

where $h_{\mathbb{D}}$ is the Koenigs map of $\varphi$.

Proof. In the rest of this proof, $D_{H}(z, r)$ will denote the open pseudo-hyperbolic disk centered at $z \in \mathbb{D}$ with radius $r \in(0,1)$. Set $c=1 / 12$.

As in Theorem 5.1, we denote by $V(\rho)=\left\{z \in \mathbb{D}: \widetilde{\rho}_{\mathbb{D}}(\varphi(z), z)<\rho\right\}$. Clearly, for every $z \in V(c / 2)$, we have that $D_{H}(z, c) \subset V(1 / 3)$. This inclusion tells us, by the former theorem, that $h_{\mathbb{D}}$ is univalent in $D_{H}(z, c)$, for all $z \in V(c / 2)$.

Fix $z \in V(c / 2)$ and consider the analytic function

$$
g_{z}(s):=\frac{h_{\mathbb{D}}\left(\frac{c s+z}{1+\bar{z} c s}\right)-h_{\mathbb{D}}(z)}{\left(1-|z|^{2}\right) c h_{\mathbb{D}}^{\prime}(z)}, \quad s \in \mathbb{D} .
$$

Note that $g_{z}$ is well defined because $\frac{c s+z}{1+\bar{z} c s} \in \mathbb{D}$ and the univalence property of $h_{\mathbb{D}}$ holds in $V(1 / 3)$. For every $s \in \mathbb{D}$, we have $\widetilde{\rho}_{\mathbb{D}}\left(z, \frac{c s+z}{1+\bar{z} c s}\right)<c$, so

$$
\left\{\frac{c s+z}{1+\bar{z} c s}: s \in \mathbb{D}\right\} \subset D_{H}(z, c)
$$

and we can assure that $g_{z}$ is univalent in $\mathbb{D}$. Clearly, $g_{z}(0)=0$ and $g_{z}^{\prime}(0)=1$. Therefore, by the Koebe Distortion Theorem [11, p. 21], we deduce that, for every $s \in \mathbb{D}$,

$$
\left|g_{z}(s)\right| \leq \frac{|s|}{(1-|s|)^{2}}
$$


Now, using the Cauchy Integral Formula and the above bound, we find that, for every $z \in V(c / 2)$ and for every $|s| \leq 1 / 2$,

$$
\begin{aligned}
\left|g_{z}(s)-s\right| & =\left|g_{z}(s)-g_{z}(0)-g_{z}^{\prime}(0) s\right| \\
& =\left|\frac{1}{2 \pi i} \int_{C^{+}(0,2 / 3)} g_{z}(\xi)\left(\frac{1}{\xi-s}-\frac{1}{\xi}-\frac{s}{\xi^{2}}\right) d \xi\right| \\
& =\left|\frac{1}{2 \pi i} \int_{C^{+}(0,2 / 3)} g_{z}(\xi) \frac{s^{2}}{\xi^{2}(\xi-s)} d \xi\right| \\
& \leq \frac{1}{2 \pi} \int_{C^{+}(0,2 / 3)} \frac{|\xi|}{(1-|\xi|)^{2}} \frac{|s|^{2}}{|\xi|^{2}|\xi-s|}|d \xi| \leq \frac{1}{2 \pi} 2 \pi \frac{2}{3} \frac{1}{1 / 9} \frac{1}{2 / 3} \frac{1}{1 / 6}|s|^{2} \\
& =54|s|^{2} .
\end{aligned}
$$

As usual, $C^{+}(0,2 / 3)$ denotes the circle centred at zero with radius $2 / 3$ and positively oriented. Notice that, in spite of the fact that $g_{z}$ clearly depends on $z$, the above bound for $g_{z}(s)-s$ is independent of $z$. This fact will be quite important in the final part of the proof. Now, set $K=54$ and take $s=\frac{1}{c} \frac{\varphi(z)-z}{1-\bar{z} \varphi(z)}$. Since $z \in V(c / 2)$, we have that $|s| \leq \frac{1}{2}$. Therefore,

$$
\begin{aligned}
\left|\frac{h_{\mathbb{D}}(\varphi(z))-h_{\mathbb{D}}(z)}{\left(1-|z|^{2}\right) c h_{\mathbb{D}}^{\prime}(z)}-\frac{1}{c} \frac{\varphi(z)-z}{1-\bar{z} \varphi(z)}\right| & =\left|g_{z}(s)-s\right| \\
& \leq K \frac{1}{c^{2}}\left|\frac{\varphi(z)-z}{1-\bar{z} \varphi(z)}\right|^{2} .
\end{aligned}
$$

Since $h_{\mathbb{D}}(\varphi(z))=h_{\mathbb{D}}(z)+1$, we obtain

$$
\left|\frac{1}{(\varphi(z)-z) h_{\mathbb{D}}^{\prime}(z)}-\frac{1-|z|^{2}}{1-\bar{z} \varphi(z)}\right| \leq \frac{K}{c} \widetilde{\rho}_{\mathbb{D}}(\varphi(z), z) \frac{\left(1-|z|^{2}\right)}{|1-\bar{z} \varphi(z)|} .
$$

Moreover,

$$
\begin{aligned}
\left|\frac{1}{(\varphi(z)-z) h_{\mathbb{D}}^{\prime}(z)}-1\right| & \leq\left|1-\frac{1-|z|^{2}}{1-\bar{z} \varphi(z)}\right|+\left|\frac{1}{(\varphi(z)-z) h_{\mathbb{D}}^{\prime}(z)}-\frac{1-|z|^{2}}{1-\bar{z} \varphi(z)}\right| \\
& \leq \widetilde{\rho}_{\mathbb{D}}(\varphi(z), z)+\frac{K}{c} \widetilde{\rho}_{\mathbb{D}}(\varphi(z), z) \frac{\left(1-|z|^{2}\right)}{|1-\bar{z} \varphi(z)|} \\
& =\widetilde{\rho}_{\mathbb{D}}(\varphi(z), z)+\frac{K}{c} \widetilde{\rho}_{\mathbb{D}}(\varphi(z), z)\left|\frac{1-\bar{z} \tau}{1-\bar{z} \varphi(z)}\right| \frac{\left(1-|z|^{2}\right)}{|1-\bar{z} \tau|} .
\end{aligned}
$$

Summing up, we have proved that for all $z \in V(c / 2)$, it follows that

$$
\left|\frac{1}{(\varphi(z)-z) h_{\mathbb{D}}^{\prime}(z)}-1\right| \leq \widetilde{\rho}_{\mathbb{D}}(\varphi(z), z)\left(1+\frac{K}{c}\left|\frac{1-\bar{z} \tau}{1-\bar{z} \varphi(z)}\right| \frac{\left(1-|z|^{2}\right)}{|1-\bar{z} \tau|}\right) .
$$

Now, fix a Stolz angle $S(\tau, \alpha)$. By Theorem $5.1(3)$, there exists $\delta>0$ such that

$$
S(\tau, \alpha) \cap\{z \in \mathbb{D}:|\tau-z|<\delta\} \subset V(c / 2) .
$$

We know that

$$
\frac{\left(1-|z|^{2}\right)}{|1-\bar{z} \tau|} \leq 2+\tan \alpha .
$$


Bearing in mind that $\varphi$ is parabolic, we can even choose $\delta$ small enough such that $\left|\frac{1-\bar{z} \tau}{1-\bar{z} \varphi(z)}\right|$ is bounded in $S(\tau, \alpha) \cap\{z \in \mathbb{D}:|\tau-z|<\delta\}$. Finally, our theorem follows from the above inequality and Lemma 3.1

\section{Second order Angular Differentiability: The Zero step CASE}

In this section, we have followed a certain parallelism with the results given in the former positive h-step section. Perhaps, the first thing we must say about parabolic functions $\varphi$ of zero h-step belonging to $C_{A}^{2}(\tau)$ ( $\tau$ is the Denjoy-Wolff point) is that both possibilities $\operatorname{Re}\left(\tau \varphi^{\prime \prime}(\tau)\right)=0$ and $\operatorname{Re}\left(\tau \varphi^{\prime \prime}(\tau)\right)>0$ can happen. For instance, consider the following two examples:

$$
\phi_{1}(w)=w+1, \phi_{2}(w)=w+i+\frac{1}{\sqrt{w}}, w \in \mathbb{H} .
$$

This is in clear contrast with the situation described in Corollary 4.2. For the sake of clearness, in what follows, we refer to the case " $\operatorname{Re}\left(\tau \varphi^{\prime \prime}(\tau)\right)>0$ " as the "certainly non-tangential" one and to the other " $\operatorname{Re}\left(\tau \varphi^{\prime \prime}(\tau)\right)=0$ " as the "indefinite" one. Our first result tries to justify these names and give some insight into this dichotomy. The first equivalence of the next theorem is proved in [2] under stronger hypotheses.

Theorem 6.1. Let $\varphi \in \operatorname{Hol}(\mathbb{D} ; \mathbb{D})$ be parabolic with Denjoy-Wolff point $\tau \in \partial \mathbb{D}$. Assume that $\varphi \in C_{A}^{2}(\tau)$. Then, the following are equivalent:

(1) $\operatorname{Re}\left(\varphi^{\prime \prime}(\tau) \tau\right)>0$ (and, therefore, $\varphi$ is of zero hyperbolic step).

(2) $\varphi^{\prime \prime}(\tau) \neq 0$ and every (resp. some) forward orbit of $\varphi$ tends non-tangentially to $\tau$.

(3) Every (resp. some) forward orbit $\left(z_{n}\right)$ of $\varphi$ tends non-tangentially to $\tau$ and

$$
\lim _{n} \frac{1-\left|z_{n}\right|^{2}}{\left|\tau-z_{n}\right|^{2}} \widetilde{\rho}_{\mathbb{D}}\left(z_{n+1}, z_{n}\right)
$$

exists and is positive and finite.

Proof. As usual, $\phi \in \operatorname{Hol}(\mathbb{H} ; \mathbb{H})$ will denote the iteration map associated with $\varphi$ in $\mathbb{H}$. Since $\varphi \in C_{A}^{2}(\tau)$, we see by Proposition 2.1 that

$$
\phi(w)=w+a+\gamma(w), w \in \mathbb{H}
$$

where $a=\varphi^{\prime \prime}(\tau) \tau \in \mathbb{C}$ and $\gamma \in \operatorname{Hol}(\mathbb{H} ; \mathbb{C})$ with $\angle \lim _{w \rightarrow \infty} \gamma(w)=0$.

$(1) \Rightarrow(2)$ Clearly $\varphi^{\prime \prime}(\tau) \neq 0$. It remains to find a concrete forward orbit of $\phi$ tending non-tangentially to $\infty$ [5, Lemma 2.2]. By hypothesis, Rea $>0$, so we can find $\theta \in\left(0, \frac{\pi}{2}\right)$ such that

$$
\tan (\theta)=\beta>\frac{|\operatorname{Im} a|}{\operatorname{Re} a} \geq 0
$$

and consider the (closed) Stolz angle with respect to $\infty$ defined as

$$
S:=\left\{w \in \mathbb{H}:\left|\frac{\operatorname{Im} w}{\operatorname{Re} w}\right| \leq \beta\right\} .
$$


Since $\angle \lim _{w \rightarrow \infty} \gamma(w)=0$ and $a+\gamma \in \operatorname{Hol}(\mathbb{H} ; \mathbb{H})$, it is possible to obtain $R_{0}>0$ such that for every $w \in S$ and $\operatorname{Re} w \geq R_{0}$, we have that

$$
\left|\frac{\operatorname{Im}(a+\gamma(w))}{\operatorname{Re}(a+\gamma(w))}\right|<\beta \text {. }
$$

Let us show that $\phi(\Delta) \subset \Delta$, where $\Delta:=S \cap\left\{w \in \mathbb{H}: \operatorname{Re} w \geq R_{0}\right\}$. To that aim, take $w \in \Delta$. Since $\operatorname{Re} \phi(w) \geq \operatorname{Re} w \geq R_{0}$,

$$
\left|\frac{\operatorname{Im} \phi(w)}{\operatorname{Re} \phi(w)}\right| \leq \frac{|\operatorname{Im}(w)|+|\operatorname{Im}(a+\gamma(w))|}{\operatorname{Re} \phi(w)} \leq \frac{\beta \operatorname{Re} w+\beta \operatorname{Re}(a+\gamma(w))}{\operatorname{Re} \phi(w)}=\beta \frac{\operatorname{Re} \phi(w)}{\operatorname{Re} \phi(w)}=\beta .
$$

Therefore, taking into account that $R_{0} \in \mathbb{H}$ and $\Delta$ is $\phi$-invariant, we see that the orbit $\left(\phi_{n}\left(R_{0}\right)\right)$ is contained in $S$, so it converges non-tangentially to $\infty$.

$(2) \Rightarrow(1)$ Take an arbitrary orbit $\left(w_{n}\right):=\left(\phi_{n}\left(w_{0}\right)\right)$ of $\phi$. By hypothesis, we know that $\left(w_{n}\right)$ tends non-tangentially to $\infty$ and therefore $\lim _{n \rightarrow \infty} \gamma\left(w_{n}\right)=0$. Moreover,

$$
w_{n+1}=w_{n}+a+\gamma\left(w_{n}\right), n \in \mathbb{N} .
$$

Hence

$$
\frac{w_{n}}{n}=\frac{w_{0}}{n}+a+\frac{1}{n} \sum_{j=1}^{n} \gamma\left(w_{j-1}\right)
$$

Applying the Cesaro-limit criterion, we obtain that

$$
\lim _{n} \frac{w_{n}}{n}=a .
$$

We also have that $a \neq 0$ and always $\operatorname{Re} a \geq 0$, so we deduce that

$$
\operatorname{Arg} w_{n}=\operatorname{Arg} \frac{w_{n}}{n} \stackrel{n}{\rightarrow} \operatorname{Arg} a .
$$

But $\left(w_{n}\right)$ tends non-tangentially to $\infty$; thus $\operatorname{Arg} a \in\left(-\frac{\pi}{2}, \frac{\pi}{2}\right)$ and this obviously implies that $\operatorname{Re} a=\operatorname{Re}\left(\varphi^{\prime \prime}(\tau) \tau\right)>0$.

$(2) \Rightarrow(3)$ Let $\left(w_{n}\right)=:\left(\phi_{n}\left(w_{0}\right)\right)$ be an arbitrary orbit of $\phi$ and consider $\left(z_{n}\right):=$ $\left(\varphi_{n}\left(z_{0}\right)\right)$, the associated forward orbit of $\varphi$. Again according to [5. Lemma 2.2], we know that $\left(w_{n}\right)$ tends non-tangentially to $\infty$ and so we have $\lim _{n} \gamma\left(w_{n}\right)=0$. Then, following arguments similar to those given in $(2) \Rightarrow(1)$, it is easy to check that $\lim _{n} \frac{w_{n}}{n}=a \neq 0$ and $\lim _{n} \frac{\operatorname{Re} w_{n}}{n}=\operatorname{Re} a \geq 0$. If Re $a$ were zero, we would have that $\lim _{n}\left|\frac{\operatorname{Im} w_{n}}{\operatorname{Re} w_{n}}\right|=+\infty$ and this would contradict that $\left(w_{n}\right)$ tends non-tangentially to $\infty$. Therefore, $\operatorname{Re} a>0$ and we deduce that $\lim _{n} \operatorname{Re} w_{n}=+\infty$. Now, let us compute with the pseudo-hyperbolic distance in $\mathbb{H}$. Namely, using that $a \neq 0$,

$$
\left(\operatorname{Re} w_{n}\right) \widetilde{\rho}_{\mathbb{H}}\left(w_{n}, w_{n+1}\right)=\left|\left(a+\gamma\left(w_{n}\right)\right)\left(2+\frac{a+\gamma\left(w_{n}\right)}{\operatorname{Re} w_{n}}\right)^{-1}\right| \stackrel{n}{\longrightarrow}\left|\frac{a}{2}\right|>0 .
$$

Therefore, the sequences $\left(\widetilde{\rho}_{\mathbb{H}}\left(w_{n}, w_{n+1}\right)\right)$ and $\left(\frac{1}{\operatorname{Re} w_{n}}\right)$ are equivalent sequences as $n$ goes to $\infty$.

$(3) \Rightarrow(2)$ We only have to show that $a \neq 0$. Suppose on the contrary that $a=0$ and take an arbitrary orbit $\left(w_{n}\right)=:\left(\phi_{n}\left(w_{0}\right)\right)$ of $\phi$. Denote $x_{n}:=\operatorname{Re} w_{n}$. It is known that always $\lim _{n} x_{n} \in(0,+\infty]$. By hypothesis, all the forward orbits of $\phi$ tend nontangentially to $\infty$, so, according to [9, Remark 1], $\phi$ is necesarily of zero h-step. 
Combining this fact with

$$
\lim _{n} x_{n} \widetilde{\rho}_{\mathbb{H}}\left(w_{n}, w_{n+1}\right)>0
$$

we deduce that $\lim _{n} x_{n}=+\infty$. Since $a=0$ and using that $\lim _{n} \gamma\left(w_{n}\right)=0$, we have

$$
\lim _{n} x_{n} \widetilde{\rho}_{\mathbb{H}}\left(w_{n}, w_{n+1}\right)=\lim _{n}\left|\frac{2}{\gamma\left(w_{n}\right)}+\frac{1}{x_{n}}\right|^{-1}=0
$$

and we obtain a contradiction.

As we have just seen, in the "certainly non-tangential" case, the standard defining limit for the zero/positive h-step goes to zero in a specific quantified way. It is worth mentioning that, in the "indefinite" case, we can find orbits tending tangentially and orbits tending non-tangentially. A priori, there is a wide range of possibilities.

In our next result, we characterize being of angular-class of order two at DenjoyWolff points in terms of the Koenigs map. Roughly speaking, the result says that the derivatives of these Koenigs maps have a pole of order two at that point.

Theorem 6.2. Let $\varphi \in \operatorname{Hol}(\mathbb{D} ; \mathbb{D})$ be parabolic of zero hyperbolic step and let $\tau \in \partial \mathbb{D}$ be the corresponding Denjoy-Wolff point. Then the map $\varphi$ belongs to $C_{A}^{2}(\tau)$ if and only if the angular limit $L_{1}:=\angle \lim _{z \rightarrow \tau}(z-\tau)^{2} h_{\mathbb{D}}^{\prime}(z)$ exists in $\mathbb{C}_{\infty}$ and $L_{1} \neq 0$. Moreover, when this equivalence holds, we have $L_{1}=2 / \varphi^{\prime \prime}(\tau)$.

Assuming that $\varphi^{\prime \prime}(\tau) \neq 0$, it is also true that $\varphi$ belongs to $C_{A}^{2}(\tau)$ if and only if the angular limit $L_{2}=\angle \lim _{z \rightarrow \tau}(z-\tau) h_{\mathbb{D}}(z)$ exists finitely and $L_{2} \neq 0$. In this case, $L_{1}=-L_{2}$.

Proof. First of all, we recall that $\varphi \in C_{A}^{2}(\tau)$ if and only if the following angular limit exists finitely:

$$
\angle \lim _{z \rightarrow \tau} \frac{\varphi(z)-z}{(z-\tau)^{2}} .
$$

In fact, this limit (if it exists) is $\frac{\varphi^{\prime \prime}(\tau)}{2}$. Therefore, the first equivalence follows directly from Theorem 5.2 .

So, assume that $\varphi^{\prime \prime}(\tau) \neq 0$ and the existence of the limit $\angle \lim _{z \rightarrow \tau}(z-\tau) h_{\mathbb{D}}(z) \in$ $\mathbb{C} \backslash\{0\}$. By [12, Proposition 4.8], we obtain that $\angle \lim _{z \rightarrow \tau}(z-\tau) f^{\prime}(z)=0$, where $f(z)=(z-\tau) h_{\mathbb{D}}(z)$. That is,

$$
\angle \lim _{z \rightarrow \tau}\left[(z-\tau) h_{\mathbb{D}}(z)+(z-\tau)^{2} h_{\mathbb{D}}^{\prime}(z)\right]=0,
$$

and, therefore, $\angle \lim _{z \rightarrow \tau}(z-\tau)^{2} h_{\mathbb{D}}^{\prime}(z)=-\angle \lim _{z \rightarrow \tau}(z-\tau) h_{\mathbb{D}}(z)$.

Now, assume that $\varphi^{\prime \prime}(\tau) \neq 0$ and the existence of the limit $\angle \lim _{z \rightarrow \tau}(z-\tau)^{2} h_{\mathbb{D}}^{\prime}(z) \in$ $\mathbb{C} \backslash\{0\}$. Let us write $(z-\tau)^{2} h_{\mathbb{D}}^{\prime}(z)=b+g(z)$ with $b \in \mathbb{C} \backslash\{0\}$ and $g \in \operatorname{Hol}(\mathbb{D} ; \mathbb{C})$ with $\angle \lim _{z \rightarrow \tau} g(z)=0$. Then we have

$$
h_{\mathbb{D}}^{\prime}(z)=\frac{b}{(z-\tau)^{2}}+\frac{g(z)}{(z-\tau)^{2}}
$$

and, therefore,

$$
h_{\mathbb{D}}(z)=b-\frac{b}{z-\tau}+\int_{0}^{z} \frac{g(\xi)}{(\xi-\tau)^{2}} d \xi, \text { for every } z \in \mathbb{D} .
$$


Clearly, to finish the proof, we only have to check that $\angle \lim _{z \rightarrow \tau}(z-\tau) \int_{0}^{z} \frac{g(\xi)}{(z-\xi)^{2}} d \xi=0$. Fix $\varepsilon>0$ and a Stolz angle $\Delta=\{z \in \mathbb{D}:|\operatorname{Arg}(1-\bar{\tau} z)|<\alpha\}, 0<\alpha<\pi / 2$. This non-tangential approach to $\tau$ allows us to assure that there exists $\delta_{1}>0$ and $c>0$ such that

$$
\frac{|1-\bar{\tau} z|}{1-|z|}<c, \text { for all } z \in \Delta \text { with } 0<|z-\tau|<\delta_{1} .
$$

Moreover, since $\lim _{z \rightarrow \tau, z \in \Delta} g(z)=0$, there exists $\delta_{2} \in(0,1)$, such that $|g(z)| \leq \frac{\varepsilon}{2 c}$, for all $z \in \Delta$ with $0<|z-\tau|<\delta_{2}$. This convergence to zero also implies that there exists $M>0$, such that

$$
\sup \{|g(z)|: z \in \Delta \cap\{z \in \mathbb{D}:|z-\tau|<1\}\} \leq M .
$$

Now, for every $z \in \Delta \cap\left\{z \in \mathbb{D}:|z-\tau|<\frac{\delta_{2}}{2}\right\}$, we consider the point $P(z) \in \mathbb{D}$ obtained as the intersection between the segment $[0, z]$ and the circle of center $\tau$ and radius $\delta_{2}$. Clearly, $P(z)=t(z) z$, for some number $t(z) \in(0,1)$. It is not difficult to see that

$$
\beta:=\sup \left\{t(z): z \in \Delta \cap\left\{z \in \mathbb{D}:|z-\tau|<\frac{\delta_{2}}{2}\right\}\right\}<1 .
$$

With these elements, we define

$$
\delta:=\min \left\{\delta_{1}, \delta_{2}, \varepsilon \frac{1-\beta}{2 M}\right\}>0 .
$$

Therefore, we assume that $z \in \Delta \cap\{z \in \mathbb{D}:|z-\tau|<\delta\}$ and compute. Namely,

$$
\begin{aligned}
& \left|(\tau-z) \int_{0}^{z} \frac{g(\xi)}{(\xi-\tau)^{2}} d \xi\right| \\
\leq & |z(\tau-z)|\left|\int_{0}^{1} \frac{g(t z)}{(t z-\tau)^{2}} d t\right| \leq|z(\tau-z)| \int_{0}^{1} \frac{|g(t z)|}{|1-t z \bar{\tau}|^{2}} d t \\
\leq & |z(\tau-z)|\left[M \int_{0}^{t(z)} \frac{d t}{(1-t|z \bar{\tau}|)^{2}} d t+\frac{\varepsilon}{2 c} \int_{t(z)}^{1} \frac{d t}{(1-t|z \bar{\tau}|)^{2}} d t\right] \\
\leq & |z(\tau-z)|\left[M \frac{1}{|z|(1-t(z)|z|)}+\frac{\varepsilon}{2 c} \frac{1}{|z|(1-|z|)}\right] \\
\leq & M \frac{|\tau-z|}{1-\beta}+\frac{\varepsilon}{2 c} \frac{|\tau-z|}{1-|z|} \leq M \frac{1}{1-\beta} \frac{\varepsilon(1-\beta)}{2 M}+\frac{\varepsilon}{2 c} c=\varepsilon .
\end{aligned}
$$

Our next proposition presents the other characterization we have obtained, which deals with certain simple normalizations to obtain intertwining maps. In some sense and for zero h-step, it is the analog of Theorem 4.3 .

Proposition 6.3. Let $\varphi \in \operatorname{Hol}(\mathbb{D} ; \mathbb{D})$ be parabolic of zero hyperbolic step and with Denjoy-Wolff point $\tau \in \partial \mathbb{D}$. Likewise, let $\phi$ be the map associated with $\varphi$ in $\mathbb{H}$. Then the following are equivalent.

(1) $\varphi \in C_{A}^{2}(\tau)$ and $\operatorname{Re}\left(\tau \varphi^{\prime \prime}(\tau)\right)>0$.

(2) For some (resp. any) $w_{0} \in \mathbb{H}$, the normalized sequence

$$
g_{n}(w):=\phi_{n}(w)-\phi_{n}\left(w_{0}\right), w \in \mathbb{H}
$$


converges in the compact open topology to a non-constant function $g \in$ $\operatorname{Hol}(\mathbb{H} ; \mathbb{C})$ (of course, $g$ depends on $w_{0}$ ) such that $g \circ \phi=g+a$ with $\operatorname{Re} a>0$.

Proof. $(1) \Rightarrow(2)$ Take an arbitrary $w_{0} \in \mathbb{H}$. According to Theorem 6.1, we know that the sequence $\left(w_{n}\right)$ converges non-tangentially to $\infty$. Therefore (bearing in mind Proposition [2.1), if we write $\phi(w)=w+a+\gamma(w)$ with $\angle \lim _{w \rightarrow \infty} \gamma(w)=$ 0 , we have that $\operatorname{Re} a>0$ and $\gamma\left(\phi_{n}\left(w_{0}\right)\right)$ goes to zero as $n$ goes to $\infty$. Hence, $\phi_{n+1}\left(w_{0}\right)-\phi_{n}\left(w_{0}\right)$ converges to $a \neq 0$. By [1], we recall that the sequence of analytic functions $\left(\frac{\phi_{n}(w)-w_{n}}{w_{n+1}-w_{n}}\right)$ converges to $h$, the Koenigs map of $\phi$, where $w_{n}=\phi_{n}(1)$. Hence,

$$
\frac{\phi_{n}(w)-\phi_{n}\left(w_{0}\right)}{\phi_{n+1}\left(w_{0}\right)-\phi_{n}\left(w_{0}\right)} \stackrel{n}{\rightarrow} h(w)-h\left(w_{0}\right) .
$$

Set $g=h-h\left(w_{0}\right) \in \operatorname{Hol}(\mathbb{H} ; \mathbb{C})$. Clearly, $g \circ \phi=g+1$. Moreover,

$$
\phi_{n}(w)-\phi_{n}\left(w_{0}\right)=\left(\phi_{n+1}\left(w_{0}\right)-\phi_{n}\left(w_{0}\right)\right) \frac{\phi_{n}(w)-\phi_{n}\left(w_{0}\right)}{\phi_{n+1}\left(w_{0}\right)-\phi_{n}\left(w_{0}\right)} \stackrel{n}{\rightarrow} a g(w)
$$

uniformly on compact subsets of $\mathbb{H}$. Since Re $a>0$, the implication is proved.

$(2) \Rightarrow(1)$ Fix $w_{0} \in \mathbb{H}$ and take the corresponding $g$ and $a$. In particular, $g \circ \phi=$ $g+a$ and Re $a>0$. Note that $g\left(\phi\left(w_{0}\right)\right)=a$. In addition, write $w_{n}=\phi_{n}\left(w_{0}\right)$ and $p(w)=\phi(w)-w$. By Lemma 3.5, for every $w \in\left[w_{n}, w_{n+1}\right]$, we have that

$$
\left|p(w)-p\left(w_{n}\right)\right| \leq \frac{2 \widetilde{\rho}_{\mathbb{H}}\left(w, w_{n}\right)}{1-\widetilde{\rho}_{\mathbb{H}}\left(w, w_{n}\right)} \operatorname{Re} p\left(w_{n}\right) .
$$

Now, using (2), we deduce that $\operatorname{Re} p\left(w_{n}\right)=\operatorname{Re}\left(w_{n+1}-w_{n}\right) \stackrel{n}{\rightarrow} \operatorname{Re} g\left(\phi\left(w_{0}\right)\right)$. Note that $\operatorname{Re} g\left(\phi\left(w_{0}\right)\right) \geq 0$. At this point, we recall again that, whenever $a, b \in \mathbb{H}$, we have

$$
\max \left\{\widetilde{\rho}_{\mathbb{H}}(w, a), \widetilde{\rho}_{\mathbb{H}}(w, b)\right\} \leq \widetilde{\rho}_{\mathbb{H}}(a, b)
$$

for every $w$ belonging to the segment $[a, b]$. Therefore, for every $n$ and for every $w$ belonging to the segment $\left[w_{n}, w_{n+1}\right]$,

$$
\left|p(w)-p\left(w_{n}\right)\right| \leq \frac{2 \widetilde{\rho}_{\mathbb{H}}\left(w_{n}, w_{n+1}\right)}{1-\widetilde{\rho}_{\mathbb{H}}\left(w_{n}, w_{n+1}\right)} \operatorname{Re} p\left(w_{n}\right)
$$

But, $\varphi$ is of zero hyperbolic step; thus

$$
L=\lim _{n \rightarrow \infty} \sup _{w \in\left[w_{n}, w_{n+1}\right]}\left|p(w)-p\left(w_{n}\right)\right|=0 \cdot \operatorname{Re} g\left(w_{0}\right)=0 .
$$

Now, let us consider the polygon

$$
\Gamma:=\left[w_{0}, w_{1}\right]+\left[w_{1}, w_{2}\right]+\left[w_{2}, w_{3}\right]+\cdots .
$$

Since $\phi$ is of zero h-step, we see that $p \in \operatorname{Hol}(\mathbb{H} ; \mathbb{H})$ and we have $\operatorname{Re} w_{n+1}>\operatorname{Re} w_{n}$. So, $\Gamma$ is a Jordan arc contained in $\mathbb{H}$ with the value $\infty$ as the only $\omega$-limit. Since $L=0$, we deduce that

$$
\lim _{w \rightarrow \infty, w \in \Gamma} p(w)=\lim _{n \rightarrow \infty} p\left(w_{n}\right)=g\left(\phi\left(w_{0}\right)\right),
$$

and, by Lindelöf's Principle, we conclude that

$$
\angle \lim _{w \rightarrow \infty} p(w)=\lim _{w \rightarrow \infty, w \in \Gamma} p(w)=g\left(\phi\left(w_{0}\right)\right) .
$$


According to Proposition 2.1, this means that $\varphi \in C_{A}^{2}(\tau)$. Finally, we note that

$$
\operatorname{Re}\left(\tau \varphi^{\prime \prime}(\tau)\right)=\operatorname{Re} g\left(\phi\left(w_{0}\right)\right)=\operatorname{Re} g\left(w_{0}\right)+\operatorname{Re} a=\operatorname{Re} a>0 .
$$

Our next proposition describes an orbital estimate and a related shift type result. Among other things, this result says that arbitrary forward orbits (and also their real parts) of parabolic zero h-step tend $n$-linearly to the corresponding DenjoyWolff point in the "certainly non-tangential" case.

Proposition 6.4. Let $\varphi \in \operatorname{Hol}(\mathbb{D} ; \mathbb{D})$ be parabolic with Denjoy-Wolff point $\tau \in \partial \mathbb{D}$. Likewise, let $\phi$ be the map associated with $\varphi$ in $\mathbb{H}$. Assume that $\varphi$ belongs to $\in C_{A}^{2}(\tau)$ and $\operatorname{Re}\left(\tau \varphi^{\prime \prime}(\tau)\right)>0$ (thus, $\varphi$ is of hyperbolic zero step). Then,

(1) For some (resp. all) $z \in \mathbb{D}$, we have that

$$
\lim _{n} \frac{1}{n} \frac{1-\left|\varphi_{n}(z)\right|^{2}}{\left|1-\bar{\tau} \varphi_{n}(z)\right|^{2}}>0
$$

and

$$
\inf _{n} n \operatorname{Re}\left(1-\bar{\tau} \varphi_{n}(z)\right)>0 .
$$

(2) There exists a complex number $b$ with positive real part such that

$$
\lim _{n \rightarrow \infty} \frac{\phi_{n}(w)}{n}=b,
$$

uniformly on compact subsets of $\mathbb{H}$.

Proof. (1) Take an arbitrary orbit $\left(z_{n}\right):=\left(\varphi_{n}\left(z_{0}\right)\right)$ of $\varphi$. We note that

$$
\operatorname{Re} w_{n}=\frac{1-\left|z_{n}\right|^{2}}{\left|\tau-z_{n}\right|^{2}}, \operatorname{Im} w_{n}=\frac{2 \operatorname{Im}\left(z_{n} \bar{\tau}\right)}{\left|\tau-z_{n}\right|^{2}},
$$

where $w_{n}:=\phi_{n}\left(w_{0}\right)$ and $\sigma_{\tau}\left(z_{0}\right)=w_{0}$. By hypothesis and appealing to Theorem 6.1. we deduce that $\left(w_{n}\right)$ tends non-tangentially to $\infty$. Moreover, since $\varphi \in C_{A}^{2}(\tau)$, we see by Proposition 2.1 that

$$
\phi(w)=w+a+\gamma(w), w \in \mathbb{H},
$$

where $a=\varphi^{\prime \prime}(\tau) \tau \in \mathbb{C}$ and $\gamma \in \operatorname{Hol}(\mathbb{H} ; \mathbb{C})$ with $\angle \lim _{w \rightarrow \infty} \gamma(w)=0$. Therefore, the sequence $\left(\operatorname{Re} \gamma\left(w_{n}\right)\right)$ tends to 0 and

$$
\frac{\operatorname{Re} w_{n}}{n}=\frac{\operatorname{Re} w_{0}}{n}+\operatorname{Re} a+\frac{1}{n} \sum_{j=1}^{n} \operatorname{Re} \gamma\left(w_{j-1}\right) \stackrel{n}{\rightarrow} \operatorname{Re} a>0 .
$$

Hence, the first condition holds. Suppose now that the second condition fails. This means that there exists a subsequence $\left(n_{k}\right)$ such that

$$
\lim _{k} n_{k} \operatorname{Re}\left(1-\bar{\tau} z_{n_{k}}\right)=0 .
$$

Bearing in mind the first condition and that the sequence $\left(w_{n}\right)$ tends non-tangentially to $\infty$, we conclude that there exists $M>0$ such that, for every $n \in \mathbb{N}$,

$$
\left|\frac{\operatorname{Im} w_{n}}{n}\right|=\left|\frac{\operatorname{Im} w_{n}}{\operatorname{Re} w_{n}} \frac{\operatorname{Re} w_{n}}{n}\right| \leq M .
$$


Therefore, denoting $d_{n}:=\frac{\left|\operatorname{Im}\left(1-\bar{\tau} z_{n}\right)\right|}{\left|\operatorname{Re}\left(1-\bar{\tau} z_{n}\right)\right|}$, we have that

$$
\sup _{n} \frac{1}{n \operatorname{Re}\left(1-\bar{\tau} z_{n}\right)} \frac{d_{n}}{1+d_{n}^{2}}=\sup _{n} \frac{\left|\operatorname{Im}\left(1-\bar{\tau} z_{n}\right)\right|}{n\left|1-\bar{\tau} z_{n}\right|^{2}}=\sup _{n} \frac{\left|\operatorname{Im} w_{n}\right|}{2 n}<+\infty
$$

Necessarily, $\lim _{k} d_{n_{k}}=0$ and the sequence $\left(z_{n_{k}}\right)$ tends orthogonally to $\tau$. This also implies that

$$
\lim _{k} \frac{1-\left|z_{n_{k}}\right|}{\operatorname{Re}\left(1-\bar{\tau} z_{n_{k}}\right)}=\lim _{k} \frac{1-\left|z_{n_{k}}\right|}{\left|\tau-z_{n_{k}}\right|}=1 .
$$

Thus, again using condition one,

$$
\lim _{k} n_{k} \operatorname{Re}\left(1-\bar{\tau} z_{n_{k}}\right)=\lim _{k} n_{k}\left|\tau-z_{n_{k}}\right| \frac{\left|\tau-z_{n_{k}}\right|}{1-\left|z_{n_{k}}\right|}=2 \lim _{k} n_{k} \frac{\left|\tau-z_{n_{k}}\right|^{2}}{1-\left|z_{n_{k}}\right|^{2}}>0
$$

and we obtain a contradiction.

(2) Take the forward orbit $w_{n}:=\phi_{n}(1)$. By Theorem 6.1, we know that $\left(w_{n}\right)$ tends non-tangentially to $\infty$. Once again, using that $\varphi \in C_{A}^{2}(\tau)$ and reasoning as in the implication $(2) \Rightarrow(1)$ of Theorem 6.1, we can obtain a number $a \in \mathbb{C}$ such that $\frac{w_{n}}{n} \stackrel{n}{\rightarrow} a$. Of course, Re $a>0$. Therefore, writing $x_{n}=\operatorname{Re} \phi_{n}(1)$ and $y_{n}=\operatorname{Im} \phi_{n}(1)$, we see that

$$
\frac{x_{n}}{n} \stackrel{n}{\rightarrow} \operatorname{Re} a \text { and } \frac{y_{n}}{x_{n}}=\frac{y_{n}}{n} \frac{n}{x_{n}} \stackrel{n}{\rightarrow} \frac{\operatorname{Im} a}{\operatorname{Re} a} .
$$

At this point, according to $[9$, we recall that

$$
\frac{\phi_{n}(w)-i y_{n}}{x_{n}} \stackrel{n}{\rightarrow} 1,
$$

uniformly on compact subsets of $\mathbb{H}$. Hence,

$$
\frac{\phi_{n}(w)}{n}=\frac{x_{n}}{n}\left(\frac{\phi_{n}(w)-i y_{n}}{x_{n}}+i \frac{y_{n}}{x_{n}}\right) \stackrel{n}{\rightarrow} \operatorname{Re} a\left(1+i \frac{\operatorname{Im} a}{\operatorname{Re} a}\right)=a,
$$

uniformly on compact subsets of $\mathbb{H}$.

An immediate consequence of the above proposition is the following fact concerning slopes of forward orbits in the "certainly non-tangential" case.

Corollary 6.5. Let $\varphi \in \operatorname{Hol}(\mathbb{D} ; \mathbb{D})$ be parabolic with Denjoy-Wolff point $\tau \in \partial \mathbb{D}$. Assume that $\varphi \in C_{A}^{2}(\tau)$. If $\operatorname{Re}\left(\varphi^{\prime \prime}(\tau) \tau\right)>0$, then any forward orbit $\left(z_{n}\right)$ of $\varphi$ tends to $\tau$ with a common slope $m$. Moreover,

$$
m=\frac{\operatorname{Im}\left(\varphi^{\prime \prime}(\tau) \tau\right)}{\operatorname{Re}\left(\varphi^{\prime \prime}(\tau) \tau\right)} \in \mathbb{R}
$$

In particular, whenever $\operatorname{Im}\left(\varphi^{\prime \prime}(\tau) \tau\right)=0$, each forward orbit of $\varphi$ tends orthogonally to $\tau$.

\section{Rigidity RESUlts ON SECOND ORDER ANGULAR DERIVATIVES}

In order to achieve some information when the second angular derivative is zero, we only have to consider the results of sections three and four and negate some of their statements. We have to remember that there are examples of this kind in the positive as well as in the zero h-step. In this section, we are going to see that imposing this vanishing condition is indeed a very restricted hypothesis. This fact can be deduced from some new rigidity results that we present now and which are 
related to some results from [4, [7, [13, and [14]. We also want to point out that our technique of proof is rather different from these four cited papers.

In [13, it is proved that if $b \in \partial \mathbb{D}$ and $\varphi \in \operatorname{Hol}(\mathbb{D} ; \mathbb{D}) \cap C_{A}^{3}(b)$ satisfying that

$$
\varphi(b)=b, \varphi^{\prime}(b)=1, \varphi^{\prime \prime}(b)=0, \varphi^{\prime \prime \prime}(b)=0,
$$

then $\varphi$ is the identity in $\mathbb{D}$. The name "rigidity" becomes quite clear when thinking about how far from being true is a similar result for a fixed point of an entire or a rational function.

Our next theorem (and its proof) is the main tool for our rigidity results. For its correct understanding, we recall (using its notation) that always $\operatorname{Re}\left(\tau \varphi^{\prime \prime}(\tau)\right) \geq$ $\alpha(\alpha-1)$. So, in a certain sense, the theorem deals with an extreme situation. We also want to mention that the special case $\alpha=1$ was also considered in [7, where the assumption of the existence of the third derivative can be replaced by its finiteness.

Theorem 7.1. Let $\varphi \in \operatorname{Hol}(\mathbb{D} ; \mathbb{D})$ be non-elliptic with Denjoy-Wolff point $\tau \in \partial \mathbb{D}$ and $\alpha=\varphi^{\prime}(\tau) \in(0,1]$. Assume that $\varphi$ is not an automorphism of $\mathbb{D}$ but $\varphi \in C_{A}^{2}(\tau)$ and $\operatorname{Re}\left(\tau \varphi^{\prime \prime}(\tau)\right)=\alpha(\alpha-1)$. Then:

(1) The angular limit exists:

$$
L:=\angle \lim _{z \rightarrow \tau} \frac{\varphi(z)-\tau-\alpha(z-\tau)-\frac{\varphi^{\prime \prime}(\tau)}{2}(z-\tau)^{2}}{(z-\tau)^{3}} \in \mathbb{C}_{\infty} .
$$

That is, $\varphi^{\prime \prime \prime}(\tau):=6 L \in \mathbb{C}_{\infty}$ always exists in this generalized sense and, indeed,

$$
\frac{2}{3} \tau^{2} \varphi^{\prime \prime \prime}(\tau)-\frac{1}{\alpha}\left(\tau \varphi^{\prime \prime}(\tau)\right)^{2} \in(-\infty, 0) \cup\{\infty\} .
$$

(2) If $\varphi \in C_{A}^{3}(\tau)$ and $\left|\varphi^{\prime \prime}(\tau)\right| \geq \sqrt{2} \alpha(1-\alpha)$, then

$$
\operatorname{Re}\left(\tau^{2} \varphi^{\prime \prime \prime}(\tau)\right)<0 \text { and }\left|\varphi^{\prime \prime \prime}(\tau)\right| \geq \frac{3}{2 \alpha}\left|\varphi^{\prime \prime}(\tau)\right|^{2} .
$$

Proof. Let $\phi \in \operatorname{Hol}(\mathbb{H} ; \mathbb{H})$ be the associated analytical map with $\varphi$ in the unit disk. Since $\varphi \in C_{A}^{2}(\tau)$ and also using Proposition 2.1, we know that

$$
\phi(w)=\frac{1}{\alpha} w+a+\gamma(w), w \in \mathbb{H},
$$

where $\operatorname{Re} a \geq 0$ and $\angle \lim _{w \rightarrow \infty} \gamma(w)=0$. Moreover, using again that proposition and our hypothesis saying that $\operatorname{Re}\left(\tau \varphi^{\prime \prime}(\tau)\right)=\alpha(\alpha-1)$, we deduce that $a=i \beta$, for some $\beta \in \mathbb{R}$. Therefore, $\phi$ being non-elliptic, we see that $\operatorname{Re}(\gamma(w)) \geq 0$, for all $w \in \mathbb{H}$. Taking into account $\angle \lim _{w \rightarrow \infty} \gamma(w)=0$ and standard arguments, we find that we have the following dichotomy: $\gamma \in \operatorname{Hol}(\mathbb{H} ; \mathbb{H})$ or $\gamma$ is identically zero. In this last case, we clearly have an automorphism and so, by our hypothesis, we can assure that $\gamma \in \operatorname{Hol}(\mathbb{H} ; \mathbb{H})$ and we can define $h:=\frac{1}{\gamma}$. Moreover, since $\angle \lim _{w \rightarrow \infty} \gamma(w)=0$, we see that $h \in \operatorname{Hol}(\mathbb{H} ; \mathbb{H})$ and has $\infty$ as a (boundary) fixed point. According to the Julia-Carathéodory Theorem, the following limit exists:

$$
h^{\prime}(\infty)=\angle \lim _{w \rightarrow \infty} \frac{h(w)}{w}=\angle \lim _{w \rightarrow \infty} \frac{1}{w \gamma(w)} \in[0,+\infty) .
$$

Set $\lambda:=\frac{1}{h^{\prime}(\infty)}=\angle \lim _{w \rightarrow \infty} w \gamma(w) \in(0,+\infty) \cup\{\infty\}$. 
Since $\varphi \in C_{A}^{2}(\tau)$, we can consider

$$
q(z):=\tau+\alpha(z-\tau)+\frac{\varphi^{\prime \prime}(\tau)}{2}(z-\tau)^{2}, z \in \mathbb{D} .
$$

Beyond this point, we will make extensive usage of Propositions 2.1 and 2.2. Using the canonical change $w=\sigma_{\tau}(z)$, we deduce that

$$
\varphi(z)-q(z)=\frac{2 \tau\left(-(w+1)^{2}+\alpha(1+w)(\phi(w)+1)-\tau \varphi^{\prime \prime}(\tau)(\phi(w)+1)\right)}{(\phi(w)+1)(w+1)^{2}} .
$$

Hence,

$$
\begin{aligned}
& \frac{\varphi(z)-q(z)}{(z-\tau)^{3}}=\frac{\alpha(1+w)}{4 \tau^{2}(\phi(w)+1)}\left(-(1+w)\left(\gamma(w)+1-\frac{1}{\alpha}+\beta i\right)+\frac{\tau \varphi^{\prime \prime}(\tau)}{\alpha}(\phi(w)+1)\right) \\
& =\frac{\alpha(1+w)}{4 \tau^{2}(\phi(w)+1)}\left(-w \gamma(w)+(\alpha \beta i+\alpha-2) \gamma(w)+\frac{(\alpha-1)^{2}-\alpha^{2} \beta^{2}}{\alpha}+2 \beta(\alpha-1) i\right) .
\end{aligned}
$$

Bearing in mind that the existence of the three following limits is always guaranteed,

$$
\angle \lim _{w \rightarrow \infty} \gamma(w)=0, \angle \lim _{w \rightarrow \infty} \frac{1+w}{\phi(w)+1}=\alpha, \angle \lim _{w \rightarrow \infty} w \gamma(w)=\lambda,
$$

we deduce that the angular $\operatorname{limit} L:=\angle \lim _{z \rightarrow \tau} \frac{\varphi(z)-q(z)}{(z-\tau)^{3}}$ exists and, indeed, either $L=\infty$ (which corresponds to $\lambda=\infty$ ) or

$$
L=\frac{\alpha^{2}}{4 \tau^{2}}\left(-\lambda+\frac{(\alpha-1)^{2}-\alpha^{2} \beta^{2}}{\alpha}+2 \beta(\alpha-1) i\right) .
$$

Now, denoting $\delta:=\tau \varphi^{\prime \prime}(\tau)$, we obtain that

$$
4 \tau^{2} \frac{\varphi^{\prime \prime \prime}(\tau)}{6}=-\lambda \alpha^{2}+\frac{1}{\alpha}\left(\operatorname{Im}\left(\delta^{2}\right) i+\operatorname{Re}\left(\delta^{2}\right)\right)=-\lambda \alpha^{2}+\frac{\delta^{2}}{\alpha}
$$

and, therefore,

$$
\frac{2}{3} \tau^{2} \varphi^{\prime \prime \prime}(\tau)-\frac{1}{\alpha}\left(\tau \varphi^{\prime \prime}(\tau)\right)^{2}=-\lambda \alpha^{2} \in(-\infty, 0) .
$$

This concludes the proof of statement (1).

Now, assume that $\varphi \in C_{A}^{3}(\tau)$ and $\left|\varphi^{\prime \prime}(\tau)\right| \geq \sqrt{2} \alpha(1-\alpha)$. Since $\operatorname{Re}\left(\tau \varphi^{\prime \prime}(\tau)\right)=$ $\alpha(\alpha-1)$, we deduce that

$$
\left|\operatorname{Im}\left(\tau \varphi^{\prime \prime}(\tau)\right)\right| \geq \alpha(1-\alpha) \Leftrightarrow|\beta| \geq \frac{1-\alpha}{\alpha} .
$$

Some computations show that the meaning of this last inequality is equivalent to $\operatorname{Re}\left(\delta^{2}\right) \leq 0$. Therefore,

$$
\operatorname{Re}\left(\tau^{2} \varphi^{\prime \prime \prime}(\tau)\right)=-\frac{3}{2} \lambda \alpha^{2}+\frac{3}{2} \operatorname{Re}\left(\frac{\delta^{2}}{\alpha}\right)<0 .
$$

Finally,

$$
\left|\varphi^{\prime \prime \prime}(\tau)\right|^{2}=\left|\tau^{2} \varphi^{\prime \prime \prime}(\tau)\right|^{2} \geq \frac{9}{4} \frac{\operatorname{Re}\left(\delta^{2}\right)^{2}}{\alpha^{2}}+\frac{9}{4} \frac{\operatorname{Im}\left(\delta^{2}\right)^{2}}{\alpha^{2}}=\frac{9}{4} \frac{\left|\varphi^{\prime \prime}(\tau)\right|^{4}}{\alpha^{2}},
$$

as wanted. 
For automorphisms, the assertions given in the above theorem are clearly true. We also point out that the above statement (2) extends certain results dealing with the case that $\operatorname{Re}\left(\tau^{2} \varphi^{\prime \prime \prime}(\tau)\right)$ is negative.

Our final result is a quick consequence of our previous theorem and, at the same time, it is a particular case of a more general result by Tauraso and Vlacci [14, Theorem 2.6]. We include it for the sake of completeness and, of course, we only sketch the proof.

Proposition 7.2. Let $\varphi \in \operatorname{Hol}(\mathbb{D} ; \mathbb{D})$ be non-elliptic with Denjoy-Wolff point $\tau \in$ $\partial \mathbb{D}$ and $\alpha=\varphi^{\prime}(\tau) \in(0,1]$. Assume that $\varphi \in C_{A}^{3}(\tau)$ and $\operatorname{Re}\left(\tau \varphi^{\prime \prime}(\tau)\right)=\alpha(\alpha-1)$. Then, $S_{\varphi}(\tau)=0$ if and only if

$$
\varphi(z)=\frac{\left(\tau \varphi^{\prime \prime}(\tau)-2 \alpha^{2}\right) z+\overline{\varphi^{\prime \prime}(\tau)}}{\varphi^{\prime \prime}(\tau) z+\overline{\tau \varphi^{\prime \prime}(\tau)}-2 \alpha^{2}}, z \in \mathbb{D} .
$$

In particular:

(1) A non-trivial (i.e., $\varphi \neq i d_{\mathbb{D}}$ ) holomorphic map $\varphi \in \operatorname{Hol}(\mathbb{D} ; \mathbb{D})$ is a parabolic automorphism if and only if there exists $b \in \partial \mathbb{D}$ such that $\varphi \in C_{A}^{3}(b)$ and

$$
\varphi(b)=b, \varphi^{\prime}(b)=1, \operatorname{Re}\left(b \varphi^{\prime \prime}(b)\right)=0 \text { and } S_{\varphi}(b)=0 .
$$

(2) A holomorphic map $\varphi \in \operatorname{Hol}(\mathbb{D} ; \mathbb{D})$ is a hyperbolic automorphism if and only if there exist $b \in \partial \mathbb{D}$ and $\alpha \in(0,1)$ such that $\varphi \in C_{A}^{3}(b)$ and

$$
\varphi(b)=b, \varphi^{\prime}(b)=\alpha, \operatorname{Re}\left(b \varphi^{\prime \prime}(b)\right)=\alpha(\alpha-1) \text { and } S_{\varphi}(b)=0 .
$$

Proof. $(\Leftarrow)$ This is clear from [11, p. 176], recalling the general form of a parabolic or hyperbolic automorphism of the disk.

$(\Rightarrow)$ Assume that $S_{\varphi}(\tau)=0$. By the above theorem, $\varphi$ is an automorphism. Now, if $\phi \in \operatorname{Hol}(\mathbb{H} ; \mathbb{H})$ is the associated analytic map with $\varphi$ in the unit disk, then

$$
\phi(w)=\frac{1}{\alpha} w+\frac{\tau \varphi^{\prime \prime}(\tau)-\alpha(\alpha-1)}{\alpha^{2}}, w \in \mathbb{H} .
$$

We leave it for the reader to check that computing with the Cayley map $\sigma_{\tau}$ and its inverse, we obtain the corresponding formula for $\varphi$.

The final statement of the proposition follows easily from the first assertion, considering separately the cases $\alpha=1$ and $\alpha \in(0,1)$.

\section{ACKNOWLEDGEMENT}

We thank the referees for their valuable comments and for bringing the paper [14] to our attention.

\section{REFERENCES}

[1] I. N. Baker and Ch. Pommerenke, On the iteration of analytic functions in a halfplane. II, J. London Math. Soc. (2) 20 (1979), 255-258. MR551452 (83j:30024)

[2] P. S. Bourdon and J. H. Shapiro, Cyclic Phenomena for Composition Operators, Mem. Amer. Math. Soc. 125, Providence, 1997. MR.1396955 (97h:47023)

[3] F. Bracci and P. Poggi-Corradini, On Valiron's theorem, Future Trends in Geometric Function Theory, RNC Workshop Jyvaskyla 2003, 92 (2003), 39-55. MR2058109 (2005b:30024)

[4] D. M. Burns and S. G. Krantz, Rigidity of holomorphic mappings and a new Schwarz lemma at the boundary, J. Amer. Math. Soc. 7 (1994), 661-676. MR.1242454 (94j:32016)

[5] C. C. Cowen, Iteration and the solution of functional equations for functions analytic in the unit disk, Trans. Amer. Math. Soc. 265 (1981), 69-95. MR607108(82i:30036) 
[6] M.D. Contreras, S. Díaz-Madrigal, and Ch. Pommerenke, Some remarks on the Abel equation in the unit disk, J. London Math. Soc. 75 (2007), 623-634. MR2352725 (2008i:30025)

[7] D. Mejía and Ch. Pommerenke, The analytic fixed point function in the disk, Comput. Methods Funct. Theory 5 (2005), 275-299. MR2205415 (2006j:30006)

[8] P. Poggi-Corradini, Pointwise convergence on the boundary in the Denjoy-Wolff theorem, Preprint.

[9] Ch. Pommerenke, On the iteration of analytic functions in a halfplane, J. London Math. Soc. (2) 19 (1979), 439-447. MR540058 (83j:30023)

[10] Ch. Pommerenke, On asymptotic iteration of analytic functions in the disk, Analysis 1 (1981), 45-61. MR623642 (82g:30048)

[11] Ch. Pommerenke, Univalent Functions, Vandenhoeck \& Ruprecht, Göttingen, 1975. MR0507768 (58:22526)

[12] Ch. Pommerenke, Boundary Behaviour of Conformal Maps, Springer-Verlag, Berlin, 1992. MR1217706 (95b:30008)

[13] R. Tauraso, Commuting holomorphic self-maps of the unit disc, Ergodic Theory and Dynamical Systems 24 (2004), 945-953. MR2062926 (2005c:30022)

[14] R. Tauraso and F. Vlacci, Rigidity at the boundary for holomorphic self-maps of the unit disk, Complex Variables Theory Appl. 45 (2001), 151-165. MR 1909431(2003e:30039)

Camino de los Descubrimientos, S/n, Departamento de Matemática Aplicada iI, Escuela Técnica Superior de Ingenieros, Universidad de Sevilla, 41092, Sevilla, Spain

E-mail address: contreras@us.es

Camino de los Descubrimientos, s/n, Departamento de Matemática Aplicada iI, Escuela Técnica Superior de Ingenieros, Universidad de Sevilla, 41092, Sevilla, Spain

E-mail address: madrigal@us.es

Institut für Mathematik, Technische Universität, D-10623, Berlin, Germany

E-mail address: pommeren@math.tu-berlin.de 